\title{
Effect of epidural labor analgesia on postpartum depression and maternal and infant outcomes in parturients with gestational diabetes mellitus-A prospective cohort study
}

Gehui Li

Shenzhen maternity and child healthcare hospital

\section{Xiaofei Qi}

Shenzhen maternity and child healthcare hospital

Xuhong Tan

Shenzhen maternity and child healthcare hospital

Mingguang Wu

Shenzhen maternity and child healthcare hospital

Hao Wang

Market supervision administration of Shenzhen

\section{Xiaoguang Wang}

Shenzhen maternity and child healthcare hospital

Jing Sun

Shenzhen maternity and child healthcare hospital

\section{Yong Li}

Shenzhen maternity and child healthcare hospital

\section{Ping Wen}

Shenzhen maternity and child healthcare hospital

\section{Xiaolei Huang}

Shenzhen maternity and child healthcare hospital

Yuantao li ( $\nabla$ szmchlyt@smu.edu.cn )

Maternity and Children Hospital https://orcid.org/0000-0003-4531-7864

\section{Research article}

Keywords: epidural labor analgesia, gestational diabetes mellitus (GDM), postpartum depression (PPD), maternal and infant outcomes

Posted Date: February 12th, 2021 
DOl: https://doi.org/10.21203/rs.3.rs-228055/v1

License: (c) (1) This work is licensed under a Creative Commons Attribution 4.0 International License. Read Full License 


\section{Abstract}

\section{Background}

Postpartum depression (PPD) negatively affects the health of new mothers and can impair infant development. Both labor pain and gestational diabetes mellitus (GDM) are potential risk factors for PPD. However, the effects of epidural labor analgesia in parturients with GDM on PPD and maternal and infant outcomes have not been characterized.

Methods

Parturients with GDM in our hospital were assigned to the epidural group $(n=133)$ and no epidural (control) group ( $n=130)$ according to their choice to receive epidural labor analgesia. The Edinburgh Postnatal Depression Scale (EPDS) was used to evaluate maternal mental status at $24 \mathrm{~h}$ and 42 days postpartum. Data for relative variables in the perinatal period were collected, and the potential associations of epidural analgesia with PPD and maternal and infant outcomes were analyzed by univariate analysis and multivariate logistic regression analyses.

Results

Epidural analgesia was a protective factor for PPD at $24 \mathrm{~h}$ postpartum (odds ratio [OR], $0.301,95 \%$ confidence interval [Cl]: $0.104-0.867, \mathrm{p}<0.05$ ), and the EPDS score at $24 \mathrm{~h}$ post-delivery showed significant correlation with that at 42 days post-delivery (Pearson correlation coefficient $=0.527, p<$ 0.001). The rate of neonatal admission to the neonatal intensive care unit (NICU) for hypoglycemia was higher in the epidural group (6.92\%) than in the control group $(1.50 \% ; p<0.05)$. Epidural analgesia and drug-based diabetes control were independent predictors of the rate of neonate transfer to the NICU for hypoglycemia.

\section{Conclusion}

Epidural analgesia was a protective factor for PPD at $24 \mathrm{~h}$ postpartum, but associated with an increased risk of neonatal transfer to the NICU for hypoglycemia. Thus, monitoring of neonatal blood glucose levels after administration of epidural analgesia in parturients with GDM may be beneficial.

Clinical Trial Registration

the study was registered in the China Clinical Registration Center (URL:

http://www.chictr.org.cn/listbycreater.aspx, Registration No. ChiCTR-O0C-17013164)

\section{Background}

Gestational diabetes mellitus (GDM) refers to impaired glucose metabolism appearing during pregnancy [1] and is now one of the most common complications of pregnancy. In many countries, including 
developing countries, the morbidity of GDM has increased over $30 \%$ in the last two decades [2]. The occurrence of GDM is caused by a variety of factors and associated with increased risks of several adverse outcomes for both mothers and infants, including gestational hypertension, intrauterine death and perinatal asphyxia [3], macrosomia [4], premature delivery [5], and neonatal hypoglycemia [6]. Longterm adverse effects on mothers and infants are also possible, with research showing that parturients with GDM have an increased risk of type 2 diabetes and their offspring have an increased risk of obesity [7].

Labor pain induces a neuroendocrine response to stress that can cause a series of physiological changes, including increases in cortisol and blood glucose levels. In parturients with GDM, this can aggravate blood glucose fluctuation, causing further metabolic disorder that can have adverse effects on the fetus. Accordingly, researchers have investigated the benefits of labor analgesia in these patients and reported that it can relieve this stress response in parturients [8] and benefit the control of blood glucose and cortisol levels [9].

Postpartum depression (PPD) is a common mental disorder. Estimates of its incidence worldwide have ranged from 11-19.2\% [10-15], while estimates of its incidence in China have ranged from $6.7-34.6 \%$ [16-19]. Studies have shown that PPD can negatively affect early breastfeeding and lead to a strained mother-infant relationship $[20,21]$ and can also have detrimental effects on the long-term cognitive, social interaction, emotional, and physical development of infants [22-25]. The causes of PPD remain incompletely understood, although correlations between several factors and the occurrence of PPD have been identified. For example, the severity of pain during childbirth was shown to correlate with the occurrence of early PPD [26]. Indeed, pain often co-occurs with depression, and emerging evidence suggests an association between pain and PPD [27]. While labor pain is the main cause of adverse emotions during delivery, it remains controversial whether labor analgesia can reduce the incidence of PPD. Some studies have reported a lower incidence of PPD in patients who received epidural analgesia during delivery $[16,28,29]$, while other studies did not find that labor analgesia reduced the incidence of PPD $[9,12,30]$. O'Hara and Swain found that marital difficulties, life stress, prenatal depression, lower socioeconomic status, increased body mass index (BMI), and a history of abuse were all associated with an increased risk of PPD [11]. Multiple studies have also demonstrated that GDM is an independent risk factor for PPD [31-33]. Alterations in metabolic status, inflammation, and hypothalamus-pituitaryadrenal function may explain the potential relationship between GDM and PPD [34-36].

Although these various correlations have been reported, the effects of epidural labor analgesia on the occurrence of PPD among pregnant women with GDM as well as on maternal and infant outcomes have not been determined. Therefore, the present prospective observational cohort study was designed to investigate whether epidural labor analgesia is associated with the risk of PPD in women with GDM and to examine its impact on maternal and infant outcomes, including those related to the labor process, side effects, mode of delivery, birth details, and infant health.

\section{Methods}




\section{Study participants}

Patients were recruited in Shenzhen Maternity and Child Healthcare Hospital from January 2018 to October 2019. The inclusion criteria were: a diagnosis of GDM according to the diagnostic standard of IADPSG 2010 [37] (fasting blood glucose of 5.1-7.1 mmol/L or any blood glucose level reaching or exceeding the following criteria on a 75-g oral glucose tolerance test (OGTT) between 24 and 28 weeks of gestation: fasting blood glucose $<5.1 \mathrm{mmol} / \mathrm{L}, 1$-h blood glucose $<10.0 \mathrm{mmol} / \mathrm{L}$, and 2-h blood glucose $<$ $8.5 \mathrm{mmol} / \mathrm{L}$ ); gestational age at delivery $\geq 35$ weeks; and single fetal head position consistent with vaginal delivery. Patients were excluded if they refused participation or had: a neurological or mental disease; contraindications for intraspinal anesthesia (e.g., nerve injury symptoms during pregnancy, history of lumbar spine disease, systemic infection, and blood disease); a serious cardiovascular disease; a liver or kidney disease; or an endocrine disease other than GDM. Parturients were admitted into a delivery room when cervical dilation reached $3 \mathrm{~cm}$ for first-time mothers or $2 \mathrm{~cm}$ for mothers who had previously delivered a child. All eligible parturients were informed of the study and were included only after they provided written informed consent. Epidural labor analgesia was administered solely based upon the wishes of the parturient.

The study was conducted in accordance with the Declaration of Helsinki and Chinese clinical trial research regulations. The study protocol was approved by the Shenzhen Maternity and Child Healthcare Hospital Ethics Committee (Approval No. SZFY2017102095). Written informed consent was obtained from all enrolled parturients, and the study was registered in the China Clinical Registration Center (Registration No. ChiCTR-00C-17013164).

\section{Data collection}

The following basic demographic and clinical data were obtained for parturients by questionnaire, medical records review, and oral interview: age, height, weight, gestational week, parity, medical history (adverse pregnancy history, obstetric complications), smoking and alcohol consumption habits, long-term medication use during pregnancy, pregnancy-related information (planned or unplanned pregnancy, source of health information during pregnancy), family environment and marital status (education level of mother and father, family income, number of siblings of parturient, marital history, marital relationship during pregnancy, father's satisfaction with the infant's sex, members of the parturient's household), mental state during pregnancy, work-related information (occupation, maternity leave time, effects of childbirth on work or re-employment), and clinical details of GDM including the method of blood glucose control diabetes (diet or medication).

The following delivery-related data were recorded: administration of epidural labor analgesia, stage duration, mode of delivery, and adverse events during delivery (pruritus, dizziness, chills, nausea, vomiting, urine retention). The Numerical Rating Scale (NRS) was used to characterize pain during delivery. With this scale, the patient rates their degree of pain by reported a number from 0 to 10 , where 0 represents no pain and 10 represents the most serious pain [38]. 
The following neonatal clinical data were recorded: sex, body weight, 1- and 5-min Apgar scores, and heel blood glucose levels at 1, 2 and $3 \mathrm{~h}$ after birth. A blood glucose level less than $2.6 \mathrm{mmol} / \mathrm{l}$ is considered the limit value for clinical treatment of hypoglycemia [39]. Therefore, the blood glucose level was rechecked if $<2.6 \mathrm{mmol} / \mathrm{L}$, and a repeated abnormal level prompted transfer to the neonatal intensive care unit (NICU) for treatment of hypoglycemia. The husband's preference for the infant's sex was investigated on the second day after delivery [19].

\section{Epidural labor analgesia}

Epidural analgesia was performed at the L2-3 epidural space. After the administration of lidocaine at a test dose to exclude intravascular and subarachnoid injection, local anesthetics $(0.125 \%$ ropivacaine and $0.4 \mu \mathrm{g} / \mathrm{ml}$ sufentanil) in a 10-ml loading dose were administered. After the effect of analgesia was confirmed, a patient-controlled epidural analgesia (PCEA) pump was connected $(0.1 \%$ ropivacaine + sufentanil $0.4 \mu \mathrm{g} / \mathrm{ml}$, background dose of $5 \mathrm{ml} / \mathrm{h}$, PCEA dose of $5 \mathrm{ml} /$ bolus, locking time of $15 \mathrm{~min}$, and maximum dose of $23 \mathrm{ml} / \mathrm{h}$ ). The PCEA pump was used until delivery. If the patient reported a NRS score $>4$, an additional 5-ml bolus was administered by the anesthetic nurse. NRS scores were recorded preepidural analgesia and at cervical dilation of $5 \mathrm{~cm}$ and $10 \mathrm{~cm}$. Parturients' blood pressure was monitored, and if hypotension (decrease by $>30 \%$ baseline) appeared, norepinephrine was administered, and if bradycardia (heart rate $<60 \mathrm{bpm}$ ) occurred, atropine was administered.

\section{Postpartum assessment}

Maternal depression was assessed using the Edinburgh Postpartum Depression Scale (EDPS) at $24 \mathrm{~h}$ and 42 days after delivery $[40,41]$. In the present study, PPD was defined as a EPDS score $\geq 10$ at 42 days after delivery. The EPDS questionnaire contains 10 items, which are scored from 0 to 3 according to the severity of symptoms. The maximum total score is 30 points, and 10 points is the scoring standard for identifying PPD with great clinical significance [41]. At $24 \mathrm{~h}$ after delivery, the mental status of parturients was evaluated using the Zung Self-Rating Anxiety Scale (SAS) [42] to assess the patient's anxiety level and the Social Support Rating Scale (SSRS [43] to assess the patient's social support level. Additional information gathered during the telephone follow-up at 42 days after delivery included: the recovery of blood glucose control, the presence of chronic pain or other special conditions, the health status of the newborn, the feeding patterns of the newborn, and details of the mother's living arrangement (whether living with parent-in-law).

\section{Sample size calculation and data analysis}

The main index for the present study was the incidence of PPD at 42 days after delivery. For the sample size calculation for two independent groups, we presumed that equal numbers of patients would be assigned to the two groups, as the rate of labor analgesia use in our hospital in 2017 was $48.5 \%$. PPD was assumed as a binary result, and we assumed PPD incidence rates of $10 \%$ in the epidural labor analgesia group and of $25 \%$ in the non-epidural labor analgesia group according to the literature.[16, 28, 30] For $80 \%$ power and 0.05 two-tailed significance, the sample size needed for each group was 
calculated to be 100 by the Power Analysis and Sample Size (PASS) 2011 software (NCSS, LLC). To compensate for $20 \%$ loss to follow-up, 120 patients were needed in each group.

All statistical analyses were performed using SPSS version 25.0 software. The quantitative data were described as mean \pm standard deviation or median (quartile) according to whether the data obeyed a normal distribution, and the significance of differences between groups was determined by $t$ test or rank sum test. Counting data were described as frequency (constituent ratio), and the significance of differences between groups was determined by chi square test or Fisher exact probability test. Univariate analysis was conducted, and all factors identified as significant were included in a multivariate logistic regression analysis to analyze the relationships of 24-h and 42-day EDPS scores with epidural labor analgesia, age, BMI, SAS score, and SSRS score as well as the relationships of NICU transfer for hypoglycemia with epidural labor analgesia, age, and BMI. Epidural analgesia was set as the dependent variable, and the variables that showed a statistically significant difference between the two groups were set as independent variables. The 1:1 nearest neighbor matching method was used, and the caliper value was set to 0.02 . Propensity score matching was performed, and the matched data were analyzed by the same analysis strategy to identify significant relationships of 24-h and 42d EDPS scores and NICU transfer for hypoglycemia with other variables. For all statistical tests, significance was defined as a twotailed $\mathrm{p}$ value $<0.05$

\section{Results}

\section{Demographic and clinical characteristics of enrolled parturients}

A flow chart outlining the study enrollment process is presented in Fig. 1. Of a total of 372 eligible parturients, 68 were excluded according to the exclusion criteria. Of the 304 patients enrolled in the study, 3 patients refused participation in the 24-h follow-up, and 38 patients were lost to the 42-day follow-up. Thus, the final analysis included 263 parturients. With 130 patients in the epidural labor analgesia group (hereafter, epidural group) and 133 in the no epidural labor analgesia group (hereafter, control group), the rate of epidural labor analgesia use was $49.43 \%$.

The demographic data of the patients in each group are presented in Table 1. Patient age, history of abnormal pregnancy, and SSRS score were lower in the epidural group than in the control group (all $p<$ 0.05). Additionally, the epidural group included more primiparae and fewer multiparae compared with the control group $(p=0.000)$. More women in the epidural group reported occupations of business $\backslash$ service and housewife than in the control group (both $\mathrm{p}<0.05$ ). No significant differences in height, weight, BMI, gestational age at delivery, and SAS score were observed between the groups. 
Table 1

Baseline demographic and obstetric characteristics of parturients who completed the study

\section{Characteristic}

$\begin{array}{ll}\text { Epidural } & \begin{array}{l}\text { No epidural (control) } \\ \text { group }\end{array} \\ \text { group }(n=133)\end{array}$

$30.35 \pm 3.81$

$159.08 \pm$

5.02

Ages (years)

Height (cm)

Weight $(\mathrm{kg})$

Body mass index $\left(\mathrm{kg} / \mathrm{m}^{2}\right)$

Gestational age at delivery (weeks)

$62.05 \pm 8.14$

$24.50 \pm 2.89$

$24.24 \pm 3.97$

$39.12 \pm 0.86$

$38.94 \pm 0.96$

0.110

Gravidity

Primipara

Multipara

Parental education

Maternal education $>12 y$

Husband education $>12 y$

Family income ( $¥ / \mathrm{mo})$ a

$<3000$

$3000-6000$

6000-10000

10000-20000

$>20000$
$87(66.92 \%) \quad 31(23.31 \%)$

0.000

43(33.08\%) 102(76.69\%)

0.000

Data are presented as mean $\pm S D$, number of patients (percentage), or median (range).BMI: body mass index; SAS: Self-Rating Anxiety Scale; SSRS: Social Support Rating Scale; SD: standard deviation; $¥=$ Chinese Yuan.

${ }^{a}$ Total income of husband and wife.

${ }^{b}$ Abnormal pregnancy included embryo termination, fetal malformation, stillbirth, stillbirth history, postpartum hemorrhage, ectopic pregnancy, etc.

cObstetric diseases included pregnancy-induced hypertension syndrome, low free triiodothyronine and free thyroxine during pregnancy.

Comparisons were made using Student's t-test or Wilcoxon rank sum test for non-normally distributed variables or using Pearson's chi-squared test and Fisher's exact test for proportions. 
Characteristic

Epidural
group

No epidural (control)

group $(n=133)$

p

$(n=130)$

Occupation

Office worker

$50(38.46 \%) \quad 51(38.35 \%)$

1.000

Professional

$14(10.77 \%) \quad 13(9.77 \%)$

0.841

Business\service

$10(7.69 \%) \quad 0(0 \%)$

0.001

Self-employed

$8(6.15 \%)$

$18(13.53 \%)$

0.062

Migrant worker

$8(6.15 \%)$

$10(7.52 \%)$

0.808

Housewife

15(11.54\%) 4(3.01\%)

0.008

Others

$25(19.23 \%) \quad 37(27.82 \%)$

0.111

Source of health knowledge during pregnancy

Routine obstetric examination

$127(97.69 \quad 132(99.25 \%)$

0.366

Maternity classes

$99(76.15 \%) \quad 100(75.19 \%)$

0.886

Internet resources or books

124(95.38\%) 128(96.24\%)

0.767

Maternity leave time

None

$11(8.46 \%)$

$9(6.77 \%)$

0.648

Legal time

101(77.69\%) 114(85.71\%)

0.111

Full-time

$18(13.85 \%) \quad 10(7.52 \%)$

0.112

Maternal situational factors

Childbirth affects work or re-employment

$19(14.62 \%) \quad 14(10.53 \%)$

0.355

Data are presented as mean $\pm S D$, number of patients (percentage), or median (range).BMI: body mass index; SAS: Self-Rating Anxiety Scale; SSRS: Social Support Rating Scale; SD: standard deviation; $¥=$ Chinese Yuan.

${ }^{a}$ Total income of husband and wife.

${ }^{b}$ Abnormal pregnancy included embryo termination, fetal malformation, stillbirth, stillbirth history, postpartum hemorrhage, ectopic pregnancy, etc.

cObstetric diseases included pregnancy-induced hypertension syndrome, low free triiodothyronine and free thyroxine during pregnancy.

Comparisons were made using Student's t-test or Wilcoxon rank sum test for non-normally distributed variables or using Pearson's chi-squared test and Fisher's exact test for proportions. 


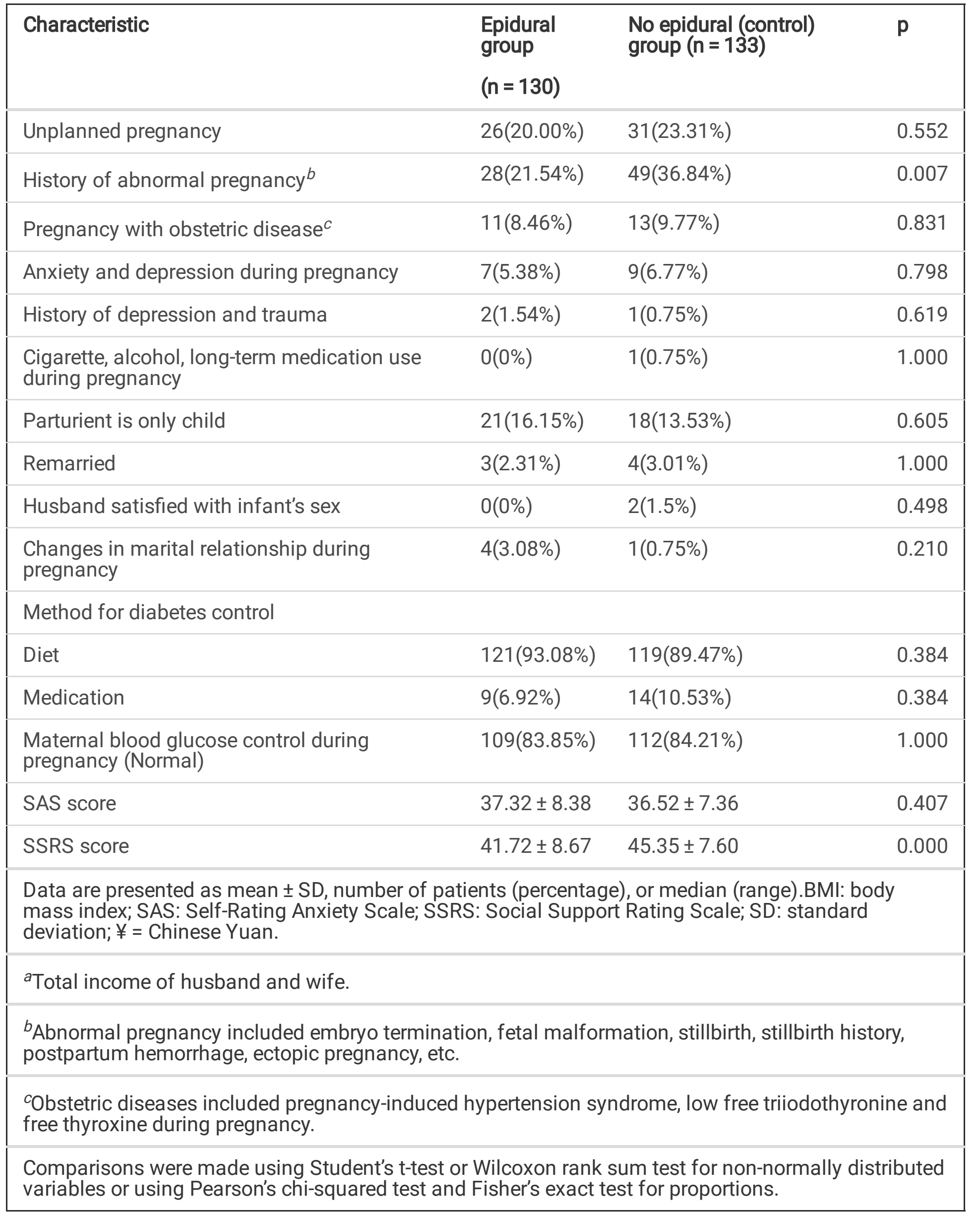


Effect of epidural labor analgesia on the occurrence of PPD, labor pain, adverse effects during delivery, and the rate of vaginal delivery

The incidence of an EDPS score at $24 \mathrm{~h}$ after delivery of $\geq 10$ was $15.38 \%(20 / 130)$ in the epidural group and $18.80 \%(25 / 133)$ the in the control group, and no significance difference was detected between the groups $(p=0.463)$. An EDPS score $\geq 10$ was detected in two more patients in the epidural group at 42 days after delivery than at $24 \mathrm{~h}$ post-delivery $(22 / 130,16.92 \%)$, whereas an EDPS score $\geq 10$ was detected in 10 fewer patients in the control group at 42 days compared with $24 \mathrm{~h}$ post-delivery $(15 / 133$, $11.28 \%$ ). Still at 42 days after delivery, the incidence of an EDPS score $\geq 10$ did not differ significantly between the two groups $(p=0.284)$. A significant correlation was found between the EPDS score at $24 \mathrm{~h}$ postpartum and that at 42 days after delivery (Pearson correlation coefficient $=0.527, p<0.001$ ).

As expected, the NRS scores for pain when cervical dilation reached $5 \mathrm{~cm}$ and $10 \mathrm{~cm}$ were significantly lower in the epidural group than in the no epidural control analgesia group $(p=0.000)$. We also observed that the durations of the first and second stages of labor were longer in the epidural group than in the control group $(p=0.000)$. Regarding adverse effects, the percentages of patients who experienced itching, dizziness, and urinary retention were significantly higher in the epidural group than in the control group (Table 2). 
Table 2

Perinatal variables of parturients who completed the study

\begin{tabular}{|c|c|c|c|}
\hline Variable & $\begin{array}{l}\text { Epidural } \\
\text { group } \\
(n=130)\end{array}$ & $\begin{array}{l}\text { No epidural (control) group }(n= \\
133 \text { ) }\end{array}$ & $\mathbf{p}$ \\
\hline NRS score & $8.98 \pm 1.77$ & $8.86 \pm 1.64$ & 0.789 \\
\hline \multicolumn{4}{|l|}{ Baseline } \\
\hline At cervical dilation $5 \mathrm{~cm}$ & $3.21 \pm 1.91$ & $9.01 \pm 1.79$ & 0.000 \\
\hline At cervical dilation $10 \mathrm{~cm}$ & $3.69 \pm 1.87$ & $9.23 \pm 1.24$ & 0.000 \\
\hline Duration of labor (min) & $594.52 \pm$ & $202.46 \pm 13.99$ & 0.000 \\
\hline \multicolumn{4}{|l|}{ Stage 1} \\
\hline Stage 2 & $52.68 \pm 46.27$ & $18.98 \pm 20.13$ & 0.000 \\
\hline Stage 3 & $9.63 \pm 6.45$ & $9.46 \pm 5.80$ & 0.820 \\
\hline \multicolumn{4}{|l|}{ Adverse effects during delivery } \\
\hline Itch & $8(6.02 \%)$ & $1(0.75 \%)$ & 0.018 \\
\hline Dizzy & $24(18.46 \%)$ & $13(9.77 \%)$ & 0.043 \\
\hline Nausea & $7(5.38 \%)$ & $7(5.26 \%)$ & 0.965 \\
\hline Vomiting & $9(6.92 \%)$ & $3(2.26 \%)$ & 0.070 \\
\hline Chills & $26(20.0 \%)$ & $17(12.78 \%)$ & 0.114 \\
\hline Urinary retention & $11(8.46 \%)$ & $1(0.75 \%)$ & 0.030 \\
\hline \multicolumn{4}{|l|}{ Mode of delivery } \\
\hline Vaginal birth & $130(100 \%)$ & $131(98.5 \%)$ & 0.498 \\
\hline Cesarean & $0(0 \%)$ & $2(1.5 \%)$ & 0.498 \\
\hline EPDS score at $24 \mathrm{~h}$ after delivery & $6.24 \pm 3.38$ & $6.33 \pm 3.87$ & 0.837 \\
\hline EPDS score at $24 \mathrm{~h} \geq 10$ & $20(15.38 \%)$ & $25(18.80 \%)$ & 0.463 \\
\hline $\begin{array}{l}\text { EPDS score at } 42 \text { days after } \\
\text { delivery }\end{array}$ & $5.91 \pm 3.18$ & $5.38 \pm 3.53$ & 0.207 \\
\hline EPDS score at 42 days $\geq 10$ & $22(16.92 \%)$ & $15(11.28 \%)$ & 0.284 \\
\hline \multicolumn{4}{|c|}{ Data are presented as mean $\pm S D$, number of patients (percentage), or median (range). } \\
\hline
\end{tabular}


Vaginal delivery was achieved by all parturients in the epidural group (100\%) and by $98.5 \%$ of parturients in the control group (131/133). No significant difference in the rate of vaginal delivery was observed between the two groups ( $p=0.199$; Table 2$)$.

\section{Effect of epidural labor analgesia on neonatal variables}

When neonatal health outcomes were compared between the epidural group and no epidural control group, no significant differences in the infants' weight, sex, and 1-min and 5-min Apgar scores were observed between the groups (Table 3). However, the incidence of newborn hypoglycemia $(<2.6 \mathrm{mmol} / \mathrm{L})$ was higher in the epidural group than in the control group at both $2 \mathrm{~h}(5.38 \%$ [7/130] vs. 0.75\% [1/133], $\mathrm{p}$ $=0.035)$ and $3 \mathrm{~h}(6.92 \%$ [9/130] vs. $1.50 \%$ [2/133], $\mathrm{p}=0.028)$ after delivery. Twenty-six newborns were transferred to the NICU ( 1 for congenital malformation, 1 for congenital pericardial effusion, 9 for mild asphyxia, 1 for jaundice, 2 for low birth weight, 2 for maternal fever, 1 for macrosomia, and 9 for hypoglycemia) in the epidural group, and 14 newborns were transferred to the NICU ( 7 for mild asphyxia, 4 for low birth weight, 1 for anemia, and 2 for hypoglycemia) in no epidural control group. The percentage of newborns who required NICU care for hypoglycemia was $6.92 \%(9 / 130)$ in the epidural group compared with only $1.50 \%(2 / 133)$ in the control group $(p=0.028)$. 
Table 3

Health outcomes for newborns of parturients enrolled in this study

\begin{tabular}{|c|c|c|c|}
\hline Neonatal outcomes & $\begin{array}{l}\text { Epidural } \\
\text { group } \\
(n=130)\end{array}$ & $\begin{array}{l}\text { No epidural (control) } \\
\text { group, } \\
(n=133)\end{array}$ & p \\
\hline Neonatal weight (g) & $3279 \pm 405$ & $3189 \pm 469$ & 0.099 \\
\hline Neonatal weight $\geq 3500 \mathrm{~g}$ & $35(26.92 \%)$ & $30(22.55 \%)$ & 0.475 \\
\hline Neonatal gender & $78(60.0 \%)$ & $72(54.14 \%)$ & 0.337 \\
\hline \multicolumn{4}{|l|}{ Male } \\
\hline Female & $52(40.0 \%)$ & $61(45.86 \%)$ & 0.337 \\
\hline Apgar score & $10(7-10)$ & $10(9-10)$ & 0.983 \\
\hline \multicolumn{4}{|l|}{$1 \mathrm{~min}$} \\
\hline $5 \min$ & $10(10-10)$ & $10(10-10)$ & 0.324 \\
\hline \multicolumn{4}{|l|}{ Heel blood glucose after birth } \\
\hline$<2.6 \mathrm{mmol} / \mathrm{L}$ at $1 \mathrm{~h}$ & $15(11.54 \%)$ & $8(6.02 \%)$ & 0.115 \\
\hline$<2.6 \mathrm{mmol} / \mathrm{L}$ at $2 \mathrm{~h}$ & $8(6.15 \%)$ & $1(0.75 \%)$ & 0.035 \\
\hline$<2.6 \mathrm{mmol} / \mathrm{L}$ at $3 \mathrm{~h}$ & $7(5.38 \%)$ & $2(1.50 \%)$ & 0.028 \\
\hline NICU admission after birth & $26(20.0 \%)$ & $14(10.53 \%)$ & 0.030 \\
\hline $\begin{array}{l}\text { NICU admission after birth for hypoglycemia } \\
\text { treatment }\end{array}$ & $9(6.92 \%)$ & $2(1.50 \%)$ & 0.028 \\
\hline
\end{tabular}

\section{Factors associated with the occurrence of PPD}

When PPD at $24 \mathrm{~h}$ after delivery was used as a dependent variable, univariate logistic regression analysis identified a total of five significant maternal and neonatal variables: anxiety and depression during pregnancy, unplanned pregnancy, maternal blood glucose control during pregnancy, SAS score, and SSRS score $(p<0.05)$. Of these factors, two were identified as significantly associated with PPD at $24 \mathrm{~h}$ after delivery on multivariate logistic regression analysis, the SAS and SSRS scores at $24 \mathrm{~h}$ after delivery (Table 4). 
Table 4

Results of univariate and multivariate analyses of factors associated with PPD at $24 \mathrm{~h}$ after delivery

\section{Variable}

Independent

Epidural analgesia

General information

Age (years)

Gestational age at delivery (weeks)

$\operatorname{BMI}\left(\mathrm{kg} / \mathrm{m}^{2}\right)$

Gravidity

(Primipara vs multipara)

Maternal education $>12 y$

Husband education $>12 y$

\section{Occupation}

Office worker

Professional/business

Service

Self-employed

Migrant worker Univariate analysis $(n=$ 263)

p OR $(95 \% \mathrm{Cl})$

0.463

$0.785(0.412-$ 1.497)
Multivariate analysis $(n=263)$ p $\quad \begin{aligned} & \mathrm{OR} \\ & \mathrm{Cl})\end{aligned}$

0.338

0.688

$(0.320-$

1.479)

0.613

$1.020(0.945-$

$0.574 \quad 1.025$

1.101)

(0.940-

1.119)

$\begin{array}{ll}0.053 & 0.831(0.689- \\ & 1.003)\end{array}$

$0.393 \quad 0.959(0.871-$

0.196

0.929

1.056)

$0.916(0.481-$

1.744)
$0.689 \quad 0.861(0.415-$

1.788)

$0.335 \quad 0.695(0.332-$

1.456)

1.039)




\begin{tabular}{|c|c|c|c|c|}
\hline \multirow{2}{*}{$\begin{array}{l}\text { Variable } \\
\text { Housewife }\end{array}$} & \multicolumn{2}{|c|}{$\begin{array}{l}\text { Univariate analysis }(n= \\
263)\end{array}$} & \multicolumn{2}{|c|}{$\begin{array}{l}\text { Multivariate } \\
\text { analysis }(n=263)\end{array}$} \\
\hline & 0.590 & $\begin{array}{l}1.359(0.445- \\
4.148)\end{array}$ & & \\
\hline Others & 0.247 & $\begin{array}{l}1.661(0.703- \\
3.928)\end{array}$ & & \\
\hline \multicolumn{5}{|l|}{ Family income $(¥ / m o)$} \\
\hline$\leq 3,000$ & \multicolumn{2}{|r|}{1.000 (Ref) } & & \\
\hline $3000-6,000$ & 0.931 & $\begin{array}{l}1.111(0.103- \\
11.965)\end{array}$ & & \\
\hline $6000-10000$ & 0.466 & $\begin{array}{l}0.419(0.040- \\
4.356)\end{array}$ & & \\
\hline $10000-20000$ & 0.757 & $\begin{array}{l}0.692(0.067- \\
7.128)\end{array}$ & & \\
\hline$>20000$ & 0.644 & $\begin{array}{l}0.568(0.051- \\
6.275)\end{array}$ & & \\
\hline Impact of childbirth on work or re-employment & 0.996 & $\begin{array}{l}1.003(0.390- \\
2.578)\end{array}$ & & \\
\hline Remarried & 0.841 & $\begin{array}{l}0.803(0.094- \\
6.837)\end{array}$ & & \\
\hline History of abnormal pregnancy & 0.950 & $\begin{array}{l}0.978(0.482- \\
1.983)\end{array}$ & & \\
\hline Pregnancy with obstetric disease & 0.431 & $\begin{array}{l}0.606(0.174- \\
2.110)\end{array}$ & & \\
\hline \multicolumn{5}{|l|}{ Maternity leave time } \\
\hline None & \multicolumn{2}{|r|}{1.000 (Ref) } & & 1.000 (Ref) \\
\hline Legal time & 0.146 & $\begin{array}{l}2.491(0.729- \\
8.514)\end{array}$ & 0.097 & $\begin{array}{l}3.087 \\
(0.816- \\
11.680)\end{array}$ \\
\hline Full time & 0.038 & $\begin{array}{l}11.333(1.150- \\
111.692)\end{array}$ & 0.161 & $\begin{array}{l}5.590 \\
(0.503- \\
62.165)\end{array}$ \\
\hline Anxiety and depression during pregnancy & 0.006 & $\begin{array}{l}4.278(1.502- \\
12.181)\end{array}$ & 0.169 & $\begin{array}{l}2.276 \\
(0.704- \\
7.353)\end{array}$ \\
\hline \multicolumn{5}{|c|}{$\begin{array}{l}\text { OR: odds ratio; } 95 \% \text { Cl: } 95 \% \text { confidence interval; BMI: body mass index; SAS: Self-Rating Anxiety } \\
\text { Scale; SSRS: Social Support Rating Scale; } ¥ \text { : Chinese Yuan. Hosmer and Lemeshow goodness of fit } \\
\text { (GOF) test; } \chi^{2}=13.304, d f=8, p=0.102 \text {. Cox and Snell pseudo-R } R^{2}=0.137 \text {. Nagelkerke pseudo- } R^{2}= \\
0.228 \text {. }\end{array}$} \\
\hline
\end{tabular}




\section{Variable}

Univariate analysis $(\mathrm{n}=$

263)

Multivariate

analysis $(n=263)$

History of depression and trauma

Cigarette, alcohol, and long-term medication use

Husband's satisfaction with infant's sex

0.262

NA

NA

$0.203(0.012-$

$3.303)$

Source of health knowledge during pregnancy

Routine obstetric examination

$\begin{array}{ll}0.195 & 0.300 \text { (0.049- } \\ & 1.850)\end{array}$

Maternity classes

$\begin{array}{ll}0.985 & 0.993(0.470- \\ & 2.095)\end{array}$

Internet resources or books

Unplanned pregnancy

$\begin{array}{ll}0.923 & 0.926(0.193- \\ & 4.437)\end{array}$

0.010

$2.426(1.231-$
$4.779)$

0.113

1.905

$(0.859-$

4.223)

\begin{tabular}{|c|c|c|}
\hline Parturient is only child & 0.757 & $\begin{array}{l}0.862(0.338- \\
2.199)\end{array}$ \\
\hline Changes in marital relationship during pregnancy & 0.863 & $\begin{array}{l}1.216(0.133- \\
11.142)\end{array}$ \\
\hline Method of GDM control (diet vs. medication) & 0.952 & $\begin{array}{l}0.966(0.314- \\
2.975)\end{array}$ \\
\hline $\begin{array}{l}\text { Maternal blood glucose control during pregnancy } \\
\text { (abnormal vs. normal) }\end{array}$ & 0.003 & $\begin{array}{l}0.326(0.155- \\
0.688)\end{array}$ \\
\hline SAS score & $<.001$ & $\begin{array}{l}1.096(1.047-1 \\
.146)\end{array}$ \\
\hline SSRS score & 0.006 & $\begin{array}{l}0.949(0.914- \\
0.985)\end{array}$ \\
\hline Mode of delivery (Cesarean vs. vaginal birth) & & NA \\
\hline Neonatal weight $\geq 3500 \mathrm{~g}$ & 0.626 & $\begin{array}{l}0.827(0.384- \\
1.777)\end{array}$ \\
\hline NICU admission after birth & 0.599 & $\begin{array}{l}1.257(0.536- \\
2.944)\end{array}$ \\
\hline
\end{tabular}

OR: odds ratio; 95\% Cl: 95\% confidence interval; BMl: body mass index; SAS: Self-Rating Anxiety Scale; SSRS: Social Support Rating Scale; ¥: Chinese Yuan. Hosmer and Lemeshow goodness of fit (GOF) test; $\chi^{2}=13.304, d f=8, p=0.102$. Cox and Snell pseudo- $R^{2}=0.137$. Nagelkerke pseudo- $R^{2}=$ 0.228 . 


\begin{tabular}{|c|c|c|c|}
\hline \multirow{2}{*}{$\begin{array}{l}\text { Variable } \\
\text { NICU admission after birth for hypoglycemia } \\
\text { treatment }\end{array}$} & \multicolumn{2}{|c|}{$\begin{array}{l}\text { Univariate analysis }(n= \\
263)\end{array}$} & \multirow[t]{2}{*}{$\begin{array}{l}\text { Multivariate } \\
\text { analysis }(n=263)\end{array}$} \\
\hline & 0.923 & $\begin{array}{l}1.080(0.225- \\
5.176)\end{array}$ & \\
\hline \multicolumn{4}{|c|}{$\begin{array}{l}\text { OR: odds ratio; } 95 \% \text { Cl: } 95 \% \text { confidence interval; BMI: body mass index; SAS: Self-Rating Anxiety } \\
\text { Scale; SSRS: Social Support Rating Scale; } ¥ \text { : Chinese Yuan. Hosmer and Lemeshow goodness of fit } \\
\text { (GOF) test; } \chi^{2}=13.304, \mathrm{df}=8, p=0.102 \text {. Cox and Snell pseudo- } R^{2}=0.137 \text {. Nagelkerke pseudo- } R^{2}= \\
0.228 \text {. }\end{array}$} \\
\hline
\end{tabular}

In addition, propensity score matching was performed to reduce the potential selection bias and verify the results of the univariate and multivariate analyses with "epidural analgesia" as the dependent variable and the other factors as the independent variables. The variables that showed a statistically significant difference between the two groups were then taken as the independent variables (age, gravidity, occupation, history of adverse pregnancy outcomes, and SSRS score). The 1:1 nearest neighbor matching method was used, and the caliper value was set to 0.02 . A total of 69 fuzzy matching pairs were obtained. Logistic regression analysis was performed after matching. On univariate analysis, PPD at $24 \mathrm{~h}$ after delivery was considered the dependent variable and "epidural analgesia" and "other confounding factors" as independent variables. Then the factors for which $p<0.05$ were included in the multivariate model, and stepwise regression was performed according to the Akaike information criterion minimum standard (Table 5). From this analysis, three independent factors were identified: epidural analgesia (protective factor), unplanned pregnancy (risk factor), and SAS score (risk factor). Epidural analgesia was a protective factor against PPD at $24 \mathrm{~h}$ after delivery (odds ratio [OR], 0.301; 95\% confidence interval [Cl], 0.104-0.867; $p<0.05)$. 
Table 5

Results of univariate and multivariate analyses of factors associated with PPD at $24 \mathrm{~h}$ after delivery after propensity score matching.

\begin{tabular}{|c|c|c|c|c|}
\hline \multirow{2}{*}{$\begin{array}{l}\text { Variable } \\
\text { Independent }\end{array}$} & \multicolumn{2}{|c|}{$\begin{array}{l}\text { Univariate analysis ( } \mathrm{n} \\
=138 \text { ) }\end{array}$} & \multicolumn{2}{|c|}{$\begin{array}{l}\text { Multivariate } \\
\text { analysis }(n=138)\end{array}$} \\
\hline & $\mathbf{p}$ & OR $(95 \% \mathrm{Cl})$ & $\mathbf{p}$ & $\begin{array}{l}\mathrm{OR}(95 \% \\
\mathrm{Cl})\end{array}$ \\
\hline Epidural analgesia & 0.058 & $\begin{array}{l}0.425 \\
(0.176- \\
1.028)\end{array}$ & 0.026 & $\begin{array}{l}0.301 \\
(0.104- \\
0.867)\end{array}$ \\
\hline \multicolumn{5}{|l|}{ General information } \\
\hline Age (years) & 0.384 & $\begin{array}{l}0.953 \\
(0.856- \\
1.061)\end{array}$ & 0.707 & $\begin{array}{l}0.977 \\
(0.865- \\
1.103)\end{array}$ \\
\hline Gestational age at delivery (weeks) & 0.037 & $\begin{array}{l}0.772 \\
(0.605- \\
0.984)\end{array}$ & 0.076 & $\begin{array}{l}0.753 \\
(0.550- \\
1.031)\end{array}$ \\
\hline $\mathrm{BMI}\left(\mathrm{kg} / \mathrm{m}^{2}\right)$ & 0.399 & $\begin{array}{l}0.953 \\
(0.852- \\
1.066)\end{array}$ & 0.346 & $\begin{array}{l}0.940 \\
(0.826- \\
1.069)\end{array}$ \\
\hline $\begin{array}{l}\text { Gravidity } \\
\text { (Primipara vs multipara) }\end{array}$ & 0.749 & $\begin{array}{l}1.150 \\
(0.489- \\
2.701)\end{array}$ & & \\
\hline Maternal education $>12 y$ & 0.571 & $\begin{array}{l}0.763(0.300- \\
1.941)\end{array}$ & & \\
\hline Husband education $>12 y$ & 0.225 & $\begin{array}{l}0.554 \\
(0.214- \\
1.437)\end{array}$ & & \\
\hline \multicolumn{5}{|l|}{ Occupation } \\
\hline Office worker & & \multicolumn{3}{|l|}{1.000 (Ref) } \\
\hline Professional/business & 1.000 & \multicolumn{3}{|l|}{$\begin{array}{l}1.000 \\
(0.187- \\
5.357)\end{array}$} \\
\hline Service & 0.782 & \multicolumn{3}{|l|}{$\begin{array}{l}1.227 \\
(0.288- \\
5.226)\end{array}$} \\
\hline Self-employed & 0.417 & \multicolumn{3}{|l|}{$\begin{array}{l}0.409 \\
(0.047- \\
3.543)\end{array}$} \\
\hline \multicolumn{5}{|c|}{$\begin{array}{l}\text { Data were matched by using propensity score matching with 1:1 nearest neighbor matching. Hosme } \\
\text { and Lemeshow goodness of fit (GOF) test; } \chi^{2}=7.083, \mathrm{df}=8, p=0.528 \text {. Cox and Snell pseudo- } \mathrm{R}^{2}= \\
0.199 \text {. Nagelkerke pseudo- } \mathrm{R}^{2}=0.316 \text {. }\end{array}$} \\
\hline
\end{tabular}




\begin{tabular}{|c|c|c|c|}
\hline \multirow{2}{*}{$\begin{array}{l}\text { Variable } \\
\text { Migrant worker }\end{array}$} & \multicolumn{2}{|c|}{$\begin{array}{l}\text { Univariate analysis ( } \mathrm{n} \\
=138 \text { ) }\end{array}$} & \multirow[t]{2}{*}{$\begin{array}{l}\text { Multivariate } \\
\text { analysis }(n=138)\end{array}$} \\
\hline & 0.892 & $\begin{array}{l}1.125 \\
(0.207- \\
6.123)\end{array}$ & \\
\hline Housewife & 0.959 & $\begin{array}{l}1.038 \\
(0.248- \\
4.340)\end{array}$ & \\
\hline Others & 0.274 & $\begin{array}{l}1.929 \\
(0.595- \\
6.254)\end{array}$ & \\
\hline \multicolumn{4}{|l|}{ Family income ( $¥ / m o)$} \\
\hline$\leq 3,000$ & \multicolumn{2}{|r|}{1.000 (Ref) } & \\
\hline $3000-6,000$ & 0.671 & \multicolumn{2}{|l|}{$\begin{array}{l}0.533 \\
(0.029- \\
9.708)\end{array}$} \\
\hline $6000-10000$ & 0.288 & \multicolumn{2}{|l|}{$\begin{array}{l}0.211 \\
(0.012- \\
3.731)\end{array}$} \\
\hline $10000-20000$ & 0.213 & \multicolumn{2}{|l|}{$\begin{array}{l}0.158 \\
(0.009- \\
2.877)\end{array}$} \\
\hline$>20000$ & 0.305 & \multicolumn{2}{|l|}{$\begin{array}{l}0.211 \\
(0.011- \\
4.121)\end{array}$} \\
\hline Impact of childbirth on work or re-employment & 0.832 & \multicolumn{2}{|l|}{$\begin{array}{l}0.866 \\
(0.230- \\
3.257)\end{array}$} \\
\hline Remarried & 0.782 & \multicolumn{2}{|l|}{$\begin{array}{l}1.385 \\
(0.138- \\
13.856)\end{array}$} \\
\hline History of abnormal pregnancy & 0.390 & \multicolumn{2}{|l|}{$\begin{array}{l}0.647 \\
(0.240- \\
1.747)\end{array}$} \\
\hline Pregnancy with obstetric disease & \multicolumn{3}{|c|}{ NA } \\
\hline \multicolumn{4}{|l|}{ Maternity leave time } \\
\hline None & \multicolumn{3}{|c|}{1.000 (Ref) } \\
\hline
\end{tabular}

Data were matched by using propensity score matching with 1:1 nearest neighbor matching. Hosmer and Lemeshow goodness of fit (GOF) test; $\chi^{2}=7.083, \mathrm{df}=8, p=0.528$. Cox and Snell pseudo- $\mathrm{R}^{2}=$ 0.199. Nagelkerke pseudo- $R^{2}=0.316$. 


\begin{tabular}{|c|c|c|c|c|}
\hline \multirow{2}{*}{$\begin{array}{l}\text { Variable } \\
\text { Legal time }\end{array}$} & \multicolumn{2}{|c|}{$\begin{array}{l}\text { Univariate analysis ( } \mathrm{n} \\
=138 \text { ) }\end{array}$} & \multicolumn{2}{|c|}{$\begin{array}{l}\text { Multivariate } \\
\text { analysis }(n=138)\end{array}$} \\
\hline & 0.812 & $\begin{array}{l}1.212 \\
(0.249- \\
5.898)\end{array}$ & & \\
\hline Full time & 0.527 & $\begin{array}{l}2.500 \\
(0.146- \\
42.800)\end{array}$ & & \\
\hline Anxiety and depression during pregnancy & 0.039 & $\begin{array}{l}4.652 \\
(1.083- \\
19.978)\end{array}$ & & \\
\hline History of depression and trauma & & NA & & \\
\hline Cigarette, alcohol, and long-term medication use & & NA & & \\
\hline Husband's satisfaction with infant's sex & & NA & & \\
\hline \multicolumn{5}{|l|}{ Source of health knowledge during pregnancy } \\
\hline Routine obstetric examination & 0.782 & $\begin{array}{l}0.722 \\
(0.072- \\
7.227)\end{array}$ & & \\
\hline Maternity classes & 0.225 & $\begin{array}{l}0.554 \\
(0.214- \\
1.437)\end{array}$ & & \\
\hline Internet resources or books & 0.855 & $\begin{array}{l}1.226 \\
(0.137- \\
10.952)\end{array}$ & & \\
\hline Unplanned pregnancy & 0.001 & $\begin{array}{l}4.496 \\
(1.824- \\
11.082)\end{array}$ & 0.008 & $\begin{array}{l}4.207 \\
(1.461- \\
12.111)\end{array}$ \\
\hline Parturient is only child & 0.962 & $\begin{array}{l}0.974 \\
(0.330- \\
2.871)\end{array}$ & & \\
\hline Changes in marital relationship during pregnancy & 0.552 & $\begin{array}{l}2.096 \\
(0.183- \\
24.008)\end{array}$ & & \\
\hline Method of GDM control (diet vs. medication) & 0.505 & $\begin{array}{l}1.609 \\
(0.397- \\
6.522)\end{array}$ & & \\
\hline
\end{tabular}

Data were matched by using propensity score matching with 1:1 nearest neighbor matching. Hosmer and Lemeshow goodness of fit (GOF) test; $\chi^{2}=7.083, \mathrm{df}=8, p=0.528$. Cox and Snell pseudo- $\mathrm{R}^{2}=$ 0.199 . Nagelkerke pseudo- $R^{2}=0.316$. 


\begin{tabular}{|c|c|c|c|c|}
\hline \multirow{2}{*}{$\begin{array}{l}\text { Variable } \\
\text { Maternal blood glucose control during pregnancy } \\
\text { (abnormal vs. normal) }\end{array}$} & \multicolumn{2}{|c|}{$\begin{array}{l}\text { Univariate analysis ( } n \\
=138 \text { ) }\end{array}$} & \multicolumn{2}{|c|}{$\begin{array}{l}\text { Multivariate } \\
\text { analysis }(n=138)\end{array}$} \\
\hline & 0.011 & $\begin{array}{l}0.261 \\
(0.093- \\
0.735)\end{array}$ & 0.066 & $\begin{array}{l}0.319 \\
(0.094- \\
1.080)\end{array}$ \\
\hline SAS score & 0.004 & $\begin{array}{l}1.089 \\
(1.027- \\
1.154)\end{array}$ & 0.034 & $\begin{array}{l}1.073 \\
(1.006- \\
1.146)\end{array}$ \\
\hline SSRS score & 0.557 & $\begin{array}{l}0.986 \\
(0.939- \\
1.035)\end{array}$ & 0.999 & $\begin{array}{l}1.000 \\
(0.943- \\
1.060)\end{array}$ \\
\hline Mode of delivery (Cesarean vs. vaginal birth) & & NA & & \\
\hline Neonatal weight $\geq 3500 \mathrm{~g}$ & 0.835 & $\begin{array}{l}0.903 \\
(0.347- \\
2.348)\end{array}$ & & \\
\hline NICU admission after birth & 0.774 & $\begin{array}{l}0.842 \\
(0.261- \\
2.716)\end{array}$ & & \\
\hline $\begin{array}{l}\text { NICU admission after birth for hypoglycemia } \\
\text { treatment }\end{array}$ & 0.441 & $\begin{array}{l}0.436 \\
(0.053- \\
3.597)\end{array}$ & & \\
\hline \multicolumn{5}{|c|}{$\begin{array}{l}\text { Data were matched by using propensity score matching with 1:1 nearest neighbor matching. Hosme } \\
\text { and Lemeshow goodness of fit (GOF) test; } \chi^{2}=7.083, \mathrm{df}=8, p=0.528 \text {. Cox and Snell pseudo- } \mathrm{R}^{2}= \\
0.199 . \text { Nagelkerke pseudo- } \mathrm{R}^{2}=0.316 \text {. }\end{array}$} \\
\hline
\end{tabular}

Only the SAS score was a common independent influencing factor on PPD at $24 \mathrm{~h}$ post-delivery in the GDM population before and after propensity matching (Fig. 2).

When set PPD at 42 days post-delivery was applied as a dependent variable, four significant factors among all of the parturient and neonatal variables were identified on univariate analysis $(p<0.05)$ : anxiety and depression during pregnancy, history of depression and trauma, unplanned pregnancy, and SAS score. Of these, multivariate logistic regression analysis then identified two factors independently associated with PPD: anxiety and depression during pregnancy and unplanned pregnancy (Table 6). 
Table 6

Results of univariate and multivariate analyses of factors associated with PPD at 42 days after delivery

Variable

Independent

Epidural analgesia

General information

Age (years)

0.589

$0.977(0.897-$ 1.063)

Univariate analysis $(\mathrm{n}=$ 263)

p OR $(95 \% \mathrm{Cl})$

0.191

1.602 (0.791-

3.247)

Multivariate analysis $(n=263)$

p OR $(95 \%$

Cl)

0.126

1.878

(0.837-

4.214)

\begin{tabular}{|c|c|c|c|c|}
\hline & & $1.063)$ & & $\begin{array}{l}(0.922- \\
1.113)\end{array}$ \\
\hline Gestational age at delivery (weeks) & 0.647 & $\begin{array}{l}0.951(0.765- \\
1.181)\end{array}$ & & \\
\hline $\mathrm{BMI}\left(\mathrm{kg} / \mathrm{m}^{2}\right)$ & 0.854 & $\begin{array}{l}0.991(0.895- \\
1.096)\end{array}$ & 0.647 & $\begin{array}{l}0.973 \\
(0.866- \\
1.093)\end{array}$ \\
\hline $\begin{array}{l}\text { Gravidity } \\
\text { (Primipara vs multipara) }\end{array}$ & 0.393 & $\begin{array}{l}0.738(0.368- \\
1.481)\end{array}$ & & \\
\hline Maternal education $>12 y$ & 0.411 & $\begin{array}{l}0.724(0.336- \\
1.563)\end{array}$ & & \\
\hline Husband education $>12 y$ & 0.627 & $\begin{array}{l}0.817(0.361- \\
1.849)\end{array}$ & & \\
\hline \multicolumn{5}{|l|}{ Occupation } \\
\hline Office worker & & 1.000 (Ref) & & \\
\hline Professional/business & 0.489 & $\begin{array}{l}0.578(0.122- \\
2.732)\end{array}$ & & \\
\hline Service & 0.971 & $\begin{array}{l}0.978(0.296- \\
3.228)\end{array}$ & & \\
\hline Self-employed & 0.867 & $\begin{array}{l}1.122(0.291- \\
4.330)\end{array}$ & & \\
\hline Migrant worker & 0.944 & $\begin{array}{l}1.060(0.214- \\
5.245)\end{array}$ & & \\
\hline Housewife & 0.833 & $\begin{array}{l}0.867(0.229- \\
3.282)\end{array}$ & & \\
\hline
\end{tabular}

Hosmer and Lemeshow goodness of fit (GOF) test; $\chi^{2}=5.181, \mathrm{df}=8, \mathrm{p}=0.738$. Cox and Snell pseudo$\mathrm{R}^{2}=0.084$. Nagelkerke pseudo- $\mathrm{R}^{2}=0.152$. 


\begin{tabular}{|c|c|c|c|c|}
\hline \multirow{2}{*}{\begin{tabular}{|l|} 
Variable \\
Others
\end{tabular}} & \multicolumn{2}{|c|}{$\begin{array}{l}\text { Univariate analysis }(n= \\
\text { 263) }\end{array}$} & \multicolumn{2}{|c|}{$\begin{array}{l}\text { Multivariate analysis } \\
(n=263)\end{array}$} \\
\hline & 0.383 & $\begin{array}{l}1.506(0.600- \\
3.776)\end{array}$ & & \\
\hline \multicolumn{5}{|l|}{ Family income ( $¥ / m o)$} \\
\hline$\leq 3,000$ & & 1.000 (Ref) & & \\
\hline $3000-6,000$ & 0.545 & $\begin{array}{l}0.469(0.040- \\
5.441)\end{array}$ & & \\
\hline $6000-10000$ & 0.323 & $\begin{array}{l}0.303(0.029- \\
3.228)\end{array}$ & & \\
\hline $10000-20000$ & 0.704 & $\begin{array}{l}0.636(0.062- \\
6.577)\end{array}$ & & \\
\hline$>20000$ & 0.739 & $\begin{array}{l}0.667(0.061- \\
7.271)\end{array}$ & & \\
\hline Impact of childbirth on work or re-employment & 0.322 & $\begin{array}{l}0.535(0.155- \\
1.845)\end{array}$ & & \\
\hline Remarried & 0.987 & $\begin{array}{l}1.019(0.119- \\
8.710)\end{array}$ & & \\
\hline History of abnormal pregnancy & 0.746 & $\begin{array}{l}0.879(0.403- \\
1.917)\end{array}$ & & \\
\hline Pregnancy with obstetric disease & 0.839 & $\begin{array}{l}1.124(0.364- \\
3.469)\end{array}$ & & \\
\hline \multicolumn{5}{|l|}{ Maternity leave time } \\
\hline None & & 1.000 (Ref) & & \\
\hline Legal time & 0.280 & $\begin{array}{l}1.979(0.574- \\
6.818)\end{array}$ & & \\
\hline Full time & 0.307 & $\begin{array}{l}3.778(0.294- \\
48.505)\end{array}$ & & \\
\hline Anxiety and depression during pregnancy & 0.001 & $\begin{array}{l}5.626 \\
(1.9551- \\
16.221)\end{array}$ & 0.006 & $\begin{array}{l}4.880 \\
(1.568- \\
15.193)\end{array}$ \\
\hline History of depression and trauma & 0.039 & $\begin{array}{l}12.857(1.136- \\
145.566)\end{array}$ & 0.059 & $\begin{array}{l}11.163 \\
(0.916- \\
136.075)\end{array}$ \\
\hline Cigarette, alcohol, and long-term medication use & & NA & & \\
\hline
\end{tabular}

Hosmer and Lemeshow goodness of fit (GOF) test; $\chi^{2}=5.181, \mathrm{df}=8, \mathrm{p}=0.738$. Cox and Snell pseudo$R^{2}=0.084$. Nagelkerke pseudo- $R^{2}=0.152$. 


\section{Variable}

Univariate analysis $(\mathrm{n}=$

263)

Multivariate analysis

$(n=263)$

Husband's satisfaction with infant's sex

NA

Source of health knowledge during pregnancy

Routine obstetric examination

$0.120 \quad 0.235(0.038-$

1.459)

Maternity classes

$0.411 \quad 0.724(0.336-$

1.563)

Internet resources or books

0.690

$0.726(0.151-$

3.500)

Unplanned pregnancy

0.006

$2.752(1.335-$
$5.673)$

0.030

2.404

$(1.090-$

5.305)

Parturient is only child

$\begin{array}{ll}0.808 & 0.882 \\ & 2.424)\end{array}(0.321-$

Changes in marital relationship during pregnancy

NA

Method of GDM control (diet vs. medication)

$\begin{array}{ll}0.817 \quad 0.861 & 3.046) \\ & 3.0 .244-\end{array}$

Maternal blood glucose control during pregnancy (abnormal vs. normal)

$0.139 \quad 0.532(0.230-$

1.228)

SAS score

0.035

$1.050(1.003-$

1.099)

0.215

1.031

$(0.982-$

$1.082)$

SSRS score

0.364

$0.981(0.9$
$1.022)$

0.896

0.997

$(0.852-$

1.044)

Mode of delivery (Cesarean vs. vaginal birth)

0.199

$6.250(0.382-$

102.169)

NICU admission after birth

$\begin{array}{ll}0.854 & 1.093(0.424- \\ & 2.818)\end{array}$

NICU admission after birth for hypoglycemia treatment

0.631

$0.600(0.075-$

4.830)

Chronic pain within 42 days

$\begin{array}{ll}0.459 & 1.415(0.573- \\ & 3.492)\end{array}$

42-day maternal blood glucose

Normal

1.000 (Ref)

Hosmer and Lemeshow goodness of fit (GOF) test; $\chi^{2}=5.181, \mathrm{df}=8, \mathrm{p}=0.738$. Cox and Snell pseudo$\mathrm{R}^{2}=0.084$. Nagelkerke pseudo- $\mathrm{R}^{2}=0.152$. 


\begin{tabular}{|c|c|c|c|}
\hline \multirow{2}{*}{$\begin{array}{l}\text { Variable } \\
\text { High }\end{array}$} & \multicolumn{2}{|c|}{$\begin{array}{l}\text { Univariate analysis }(n= \\
\text { 263) }\end{array}$} & \multirow[t]{2}{*}{$\begin{array}{l}\text { Multivariate analysis } \\
(\mathrm{n}=263)\end{array}$} \\
\hline & 0.766 & $\begin{array}{l}0.793(0.172- \\
3.653)\end{array}$ & \\
\hline Not tested & 0.120 & $\begin{array}{l}1.819(0.855- \\
3.870)\end{array}$ & \\
\hline \multicolumn{4}{|l|}{ Feeding patterns } \\
\hline Breastfeeding & & 1.000 (Ref) & \\
\hline Lactancia artificia & 0.941 & $\begin{array}{l}0.972(0.457- \\
2.065)\end{array}$ & \\
\hline Mixed Feeding & 0.642 & $\begin{array}{l}0.696(0.151- \\
3.214)\end{array}$ & \\
\hline Living with parent-in-law & 0.729 & $\begin{array}{l}1.144(0.533- \\
2.456)\end{array}$ & \\
\hline \multicolumn{4}{|c|}{$\begin{array}{l}\text { Hosmer and Lemeshow goodness of fit (GOF) test; } \chi^{2}=5.181, d f=8, p=0.738 . \text { Cox and Snell pseudo- } \\
R^{2}=0.084 . \text { Nagelkerke pseudo- } R^{2}=0.152 \text {. }\end{array}$} \\
\hline
\end{tabular}

In addition, propensity score matching was applied again as described above to reduce the potential selection bias and verify the results. For univariate analysis, PPD at 42 days after delivery as the dependent variable and "epidural analgesia" and "other confounding factors" as independent variables. Then the factors for which $p<0.05$ were included in the multivariate model, which identified two independent risk factors for PPD at 42 days post-delivery: anxiety and depression during pregnancy and unplanned pregnancy (Table 7). 
Table 7

Results of univariate and multivariate analyses of factors associated with PPD at 42 days after delivery after propensity score matching

\begin{tabular}{|c|c|c|c|c|}
\hline \multirow{2}{*}{$\begin{array}{l}\text { Variable } \\
\text { Independent }\end{array}$} & \multicolumn{2}{|c|}{$\begin{array}{l}\text { Univariate analysis ( } \mathrm{n} \\
=138 \text { ) }\end{array}$} & \multicolumn{2}{|c|}{$\begin{array}{l}\text { Multivariate } \\
\text { analysis }(n=138)\end{array}$} \\
\hline & $\mathbf{p}$ & OR $(95 \% \mathrm{Cl})$ & $\mathbf{p}$ & $\begin{array}{l}\mathrm{OR}(95 \% \\
\mathrm{Cl})\end{array}$ \\
\hline Epidural analgesia & 0.642 & $\begin{array}{l}1.242(0.498- \\
3.100)\end{array}$ & 0.630 & $\begin{array}{l}1.295 \\
(0.473- \\
3.549)\end{array}$ \\
\hline \multicolumn{5}{|l|}{ General information } \\
\hline Age (years) & 0.393 & $\begin{array}{l}0.950 \\
(0.845- \\
1.068)\end{array}$ & 0.316 & $\begin{array}{l}0.934 \\
(0.818- \\
1.067)\end{array}$ \\
\hline Gestational age at delivery (weeks) & 0.661 & $\begin{array}{l}0.945 \\
(0.734- \\
1.217)\end{array}$ & & \\
\hline BMI $\left(k g / m^{2}\right)$ & 0.682 & $\begin{array}{l}0.975 \\
(0.866- \\
1.099)\end{array}$ & 0.976 & $\begin{array}{l}0.982 \\
(0.859- \\
1.124)\end{array}$ \\
\hline $\begin{array}{l}\text { Gravidity } \\
\text { (Primipara vs multipara) }\end{array}$ & 0.838 & $\begin{array}{l}0.909 \\
(0.364- \\
2.272)\end{array}$ & & \\
\hline Maternal education $>12 y$ & 0.450 & $\begin{array}{l}0.682 \\
(0.253- \\
1.840)\end{array}$ & & \\
\hline Husband education $>12 y$ & 0.830 & $\begin{array}{l}0.887 \\
(0.297- \\
2.648)\end{array}$ & & \\
\hline \multicolumn{5}{|l|}{ Occupation } \\
\hline Office worker & & 1.000 (Ref) & & \\
\hline Professional/business & & NA & & \\
\hline Service & 0.850 & $\begin{array}{l}0.852 \\
(0.162- \\
4.474)\end{array}$ & & \\
\hline Self-employed & 0.483 & $\begin{array}{l}1.704 \\
(0.384- \\
7.553)\end{array}$ & & \\
\hline
\end{tabular}

Data were matched by propensity score matching with 1:1 nearest neighbor matching. Hosmer and Lemeshow goodness of fit (GOF) test; $\chi^{2}=6.441, \mathrm{df}=8, \mathrm{p}=0.598$. Cox and Snell pseudo- $\mathrm{R}^{2}=0.116$. Nagelkerke pseudo- $R^{2}=0.199$. 


\begin{tabular}{|c|c|c|c|}
\hline \multirow{2}{*}{$\begin{array}{l}\text { Variable } \\
\text { Migrant worker }\end{array}$} & \multicolumn{2}{|c|}{$\begin{array}{l}\text { Univariate analysis ( } \mathrm{n} \\
=138 \text { ) }\end{array}$} & \multirow[t]{2}{*}{$\begin{array}{l}\text { Multivariate } \\
\text { analysis }(n=138)\end{array}$} \\
\hline & 0.612 & $\begin{array}{l}0.568 \\
(0.064- \\
5.054)\end{array}$ & \\
\hline Housewife & 0.823 & $\begin{array}{l}1.179(0.278- \\
5.000)\end{array}$ & \\
\hline Others & 0.713 & $\begin{array}{l}1.278 \\
(0.345- \\
4.726)\end{array}$ & \\
\hline \multicolumn{4}{|l|}{ Family income ( $¥ / m o)$} \\
\hline$\leq 3,000$ & \multicolumn{2}{|r|}{1.000 (Ref) } & \\
\hline $3000-6,000$ & 0.394 & \multicolumn{2}{|l|}{$\begin{array}{l}0.278 \\
(0.015- \\
5.273)\end{array}$} \\
\hline $6000-10000$ & 0.158 & \multicolumn{2}{|l|}{$\begin{array}{l}0.122 \\
(0.007- \\
2.268)\end{array}$} \\
\hline $10000-20000$ & 0.169 & \multicolumn{2}{|l|}{$\begin{array}{l}0.128 \\
(0.007- \\
2.387)\end{array}$} \\
\hline$>20000$ & 0.485 & \multicolumn{2}{|l|}{$\begin{array}{l}0.353 \\
(0.019- \\
6.569)\end{array}$} \\
\hline Impact of childbirth on work or re-employment & 0.252 & \multicolumn{2}{|l|}{$\begin{array}{l}0.298 \\
(0.037- \\
2.369)\end{array}$} \\
\hline Remarried & 0.620 & \multicolumn{2}{|l|}{$\begin{array}{l}1.794 \\
(0.178- \\
18.081)\end{array}$} \\
\hline History of abnormal pregnancy & 0.482 & \multicolumn{2}{|l|}{$\begin{array}{l}0.681 \\
(0.233- \\
1.990)\end{array}$} \\
\hline Pregnancy with obstetric disease & \multicolumn{3}{|c|}{ NA } \\
\hline \multicolumn{4}{|l|}{ Maternity leave time } \\
\hline None & \multicolumn{3}{|c|}{1.000 (Ref) } \\
\hline
\end{tabular}

Data were matched by propensity score matching with 1:1 nearest neighbor matching. Hosmer and Lemeshow goodness of fit (GOF) test; $\chi^{2}=6.441, \mathrm{df}=8, \mathrm{p}=0.598$. Cox and Snell pseudo- $\mathrm{R}^{2}=0.116$. Nagelkerke pseudo- $R^{2}=0.199$. 


\begin{tabular}{|c|c|c|c|c|}
\hline \multirow{2}{*}{$\begin{array}{l}\text { Variable } \\
\text { Legal time }\end{array}$} & \multicolumn{2}{|c|}{$\begin{array}{l}\text { Univariate analysis ( } \mathrm{n} \\
=138 \text { ) }\end{array}$} & \multicolumn{2}{|c|}{$\begin{array}{l}\text { Multivariate } \\
\text { analysis }(n=138)\end{array}$} \\
\hline & 0.479 & $\begin{array}{l}2.136 \\
(0.261- \\
17.484)\end{array}$ & & \\
\hline Full time & 0.290 & $\begin{array}{l}5.500(0.235- \\
128.968)\end{array}$ & & \\
\hline Anxiety and depression during pregnancy & 0.015 & $\begin{array}{l}6.222 \\
(1.427- \\
27.131)\end{array}$ & 0.027 & $\begin{array}{l}6.197 \\
(1.233- \\
31.149)\end{array}$ \\
\hline History of depression and trauma & & NA & & \\
\hline Cigarette, alcohol, and long-term medication use & & NA & & \\
\hline Husband's satisfaction with infant's sex & & NA & & \\
\hline \multicolumn{5}{|l|}{ Source of health knowledge during pregnancy } \\
\hline Routine obstetric examination & 0.620 & $\begin{array}{l}0.558 \\
(0.055- \\
5.620)\end{array}$ & & \\
\hline Maternity classes & 0.830 & $\begin{array}{l}0.887 \\
(0.297- \\
2.648)\end{array}$ & & \\
\hline Internet resources or books & 0.960 & $\begin{array}{l}0.946 \\
(0.105- \\
8.513)\end{array}$ & & \\
\hline Unplanned pregnancy & 0.002 & $\begin{array}{l}4.524 \\
(1.732- \\
11.816)\end{array}$ & 0.003 & $\begin{array}{l}4.837 \\
(1.695- \\
13.801)\end{array}$ \\
\hline Parturient is only child & 0.931 & $\begin{array}{l}0.949 \\
(0.292- \\
3.086)\end{array}$ & & \\
\hline Changes in marital relationship during pregnancy & & NA & & \\
\hline Method of GDM control (diet vs. medication) & 0.833 & $\begin{array}{l}1.189 \\
(0.239- \\
5.917)\end{array}$ & & \\
\hline $\begin{array}{l}\text { Maternal blood glucose control during pregnancy } \\
\text { (abnormal vs. normal) }\end{array}$ & 0.514 & $\begin{array}{l}0.668 \\
(0.199- \\
2.245)\end{array}$ & & \\
\hline
\end{tabular}

Data were matched by propensity score matching with 1:1 nearest neighbor matching. Hosmer and Lemeshow goodness of fit (GOF) test; $\chi^{2}=6.441, d f=8, p=0.598$. Cox and Snell pseudo- $R^{2}=0.116$. Nagelkerke pseudo- $\mathrm{R}^{2}=0.199$. 


\begin{tabular}{|c|c|c|c|c|}
\hline \multirow{2}{*}{$\begin{array}{l}\text { Variable } \\
\text { SAS score }\end{array}$} & \multicolumn{2}{|c|}{$\begin{array}{l}\text { Univariate analysis ( } \mathrm{n} \\
=138 \text { ) }\end{array}$} & \multicolumn{2}{|c|}{$\begin{array}{l}\text { Multivariate } \\
\text { analysis }(n=138)\end{array}$} \\
\hline & 0.173 & $\begin{array}{l}1.041 \\
(0.982- \\
1.104)\end{array}$ & 0.714 & $\begin{array}{l}1.012 \\
(0.949- \\
1.080)\end{array}$ \\
\hline SSRS score & 0.559 & $\begin{array}{l}1.016 \\
(0.962- \\
1.074)\end{array}$ & 0.232 & $\begin{array}{l}1.040 \\
(0.975- \\
1.109)\end{array}$ \\
\hline Mode of delivery (Cesarean vs. vaginal birth) & & NA & & \\
\hline Neonatal weight $\geq 3500 \mathrm{~g}$ & 0.624 & $\begin{array}{l}1.280 \\
(0.477- \\
3.433)\end{array}$ & & \\
\hline NICU admission after birth & 0.678 & $\begin{array}{l}0.758 \\
(0.205- \\
2.807)\end{array}$ & & \\
\hline $\begin{array}{l}\text { NICU admission after birth for hypoglycemia } \\
\text { treatment }\end{array}$ & & NA & & \\
\hline Chronic pain within 42 days & 0.984 & $\begin{array}{l}0.987 \\
(0.262- \\
3.720)\end{array}$ & & \\
\hline \multicolumn{5}{|l|}{ 42-day maternal blood glucose } \\
\hline Normal & & 1.000 (Ref) & & \\
\hline High & 0.865 & $\begin{array}{l}1.152 \\
(0.227- \\
5.848)\end{array}$ & & \\
\hline Not tested & 0.270 & $\begin{array}{l}1.747 \\
(0.648- \\
4.710)\end{array}$ & & \\
\hline \multicolumn{5}{|l|}{ Feeding patterns } \\
\hline Breastfeeding & & 1.000 (Ref) & & \\
\hline Lactancia artificia & 0.624 & $\begin{array}{l}1.278 \\
(0.479- \\
3.409)\end{array}$ & & \\
\hline Mixed Feeding & 0.957 & $\begin{array}{l}1.045 \\
(0.206- \\
5.318)\end{array}$ & & \\
\hline
\end{tabular}

Data were matched by propensity score matching with 1:1 nearest neighbor matching. Hosmer and Lemeshow goodness of fit (GOF) test; $\chi^{2}=6.441, \mathrm{df}=8, \mathrm{p}=0.598$. Cox and Snell pseudo- $\mathrm{R}^{2}=0.116$. Nagelkerke pseudo- $\mathrm{R}^{2}=0.199$. 


\begin{tabular}{|c|c|c|c|}
\hline \multirow{2}{*}{$\begin{array}{l}\text { Variable } \\
\text { Living with parent-in-law }\end{array}$} & \multicolumn{2}{|c|}{$\begin{array}{l}\text { Univariate analysis ( } n \\
=138)\end{array}$} & \multirow[t]{2}{*}{$\begin{array}{l}\text { Multivariate } \\
\text { analysis }(n=138)\end{array}$} \\
\hline & 0.822 & $\begin{array}{l}1.125 \\
(0.402- \\
3.145)\end{array}$ & \\
\hline \multicolumn{4}{|c|}{$\begin{array}{l}\text { Data were matched by propensity score matching with } 1: 1 \text { nearest neighbor matching. Hosmer and } \\
\text { Lemeshow goodness of fit (GOF) test; } \chi^{2}=6.441, \mathrm{df}=8, \mathrm{p}=0.598 \text {. Cox and Snell pseudo- } \mathrm{R}^{2}=0.116 \text {. } \\
\text { Nagelkerke pseudo- } \mathrm{R}^{2}=0.199 \text {. }\end{array}$} \\
\hline
\end{tabular}

Anxiety and depression during pregnancy as well as unplanned pregnancy were the common independent influencing factors for PPD at 42 days post-delivery in our GDM population before and after propensity matching (Fig. 3).

Models for predicting PPD at $24 \mathrm{~h}$ and 42 days after delivery in parturients with GDM

Two factors identified on multiple logistic regression analysis as significantly associated with PPD at 24 $\mathrm{h}$ were used in a model, and the ability of that model to predict PPD at $24 \mathrm{~h}$ after delivery in parturients with GDM was tested by receiver operating characteristic (ROC) curve analysis. The model without propensity score matching showed an area under the curve (AUC) value of 0.748 (95\% Cl: $0.660-0.835$ ) when $p<0.001$ was selected as the best diagnosis point, with a sensitivity of 0.578 , specificity of 0.844 , positive predictive value of 0.433 , and negative predictive value of 0.906 . With propensity score matching, the model showed an AUC value of 0.806 (95\% Cl: $0.718-0.895)$ when $p<0.001$ was selected as the best diagnosis point, with a sensitivity of 0.815 , specificity of 0.694 , positive predictive value of 0.393 , and negative predictive value of 0.939 (Fig. 4).

The factors identified by multiple logistic regression as significantly associated with PPD at 42 days after delivery in parturients with GDM were also combined in a model, and the ability of the model to predict PPD at 42 days post-delivery in these patients was tested. The model without propensity score matching showed an AUC value of 0.716 (95\% Cl: $0.620-0.812)$ when $p<0.001$ was selected as the best diagnosis point, with a sensitivity of 0.595 , specificity of 0.770 , positive predictive value of 0.297 , and negative predictive value of 0.921 . The model with propensity score matching showed an AUC of $0.772(95 \% \mathrm{Cl}$ : $0.655-0.890$ ), with a sensitivity of 0.818 , specificity of 0.690 , positive predictive value of 0.333 , and negative predictive value of 0.952 (Fig. 5).

\section{Factors associated with neonate transfer to the NICU for hypoglycemia treatment}

To further analyze the influence of epidural labor analgesia on the health of the newborn, we considered the rate of neonatal hypoglycemia requiring further treatment in the NICU as the risk index of neonatal hypoglycemia and regarded it as the dependent variable in univariate and multivariate analyses. Univariate logistic regression analysis identified two maternal and infant variables as significantly 
associated with the rate of neonatal hypoglycemia $(p<0.05)$ : epidural analgesia and method of diabetes control (diet vs. medication). Multivariate logistic regression analysis further identified only one independent predictor, method of diabetes control (diet vs. medication) as a risk factor (OR, $5.277 ; 95 \% \mathrm{Cl}$, $1.181-23.580 ; p<0.05)$ that increased the risk of neonate transfer to the NICU for hypoglycemia treatment (Table 8). 
Table 8

Results of univariate and multivariate analyses of factors associated with the rate of neonate transfer to the NICU for hypoglycemia treatment

\begin{tabular}{|c|c|c|c|c|}
\hline \multirow{2}{*}{$\begin{array}{l}\text { Variable } \\
\text { Independent }\end{array}$} & \multicolumn{2}{|c|}{$\begin{array}{l}\text { Univariate analysis }(n= \\
\text { 263) }\end{array}$} & \multicolumn{2}{|c|}{$\begin{array}{l}\text { Multivariate analysis }(n= \\
263)\end{array}$} \\
\hline & $\mathrm{p}$ & OR $(95 \% \mathrm{Cl})$ & $\mathrm{p}$ & OR $(95 \% \mathrm{Cl})$ \\
\hline Epidural analgesia & 0.046 & $\begin{array}{l}4.872(1.032- \\
22.999)\end{array}$ & 0.072 & $\begin{array}{l}4.478(0.877- \\
22.874)\end{array}$ \\
\hline \multicolumn{5}{|l|}{ General information } \\
\hline Age (years) & 0.136 & $\begin{array}{l}0.887(0.757- \\
1.039)\end{array}$ & 0.191 & $\begin{array}{l}0.890(0.747- \\
1.060)\end{array}$ \\
\hline Gestational age at delivery (weeks) & 0.171 & $\begin{array}{l}1.588(0.819- \\
3.079)\end{array}$ & & \\
\hline BMI $\left(\mathrm{kg} / \mathrm{m}^{2}\right)$ & 0.218 & $\begin{array}{l}1.102(0.944- \\
1.287)\end{array}$ & 0.168 & $\begin{array}{l}1.145(0.944- \\
1.389)\end{array}$ \\
\hline History of abnormal pregnancy & 0.881 & $\begin{array}{l}0.902(0.233- \\
3.495)\end{array}$ & & \\
\hline Pregnancy with obstetric disease & 0.928 & $\begin{array}{l}0.908(0.112- \\
7.391)\end{array}$ & & \\
\hline Anxiety and depression during pregnancy & 0.109 & $\begin{array}{l}3.778(0.744- \\
19.170)\end{array}$ & & \\
\hline History of depression and trauma & & NA & & \\
\hline $\begin{array}{l}\text { Cigarette, alcohol, and long-term } \\
\text { medication use }\end{array}$ & & NA & & \\
\hline \multicolumn{5}{|l|}{$\begin{array}{l}\text { Source of health knowledge during } \\
\text { pregnancy }\end{array}$} \\
\hline Routine obstetric examination & 0.117 & $\begin{array}{l}0.161(0.016- \\
1.578)\end{array}$ & & \\
\hline Maternity classes & 0.108 & $\begin{array}{l}0.367(0.108- \\
1.245)\end{array}$ & & \\
\hline Internet resources or books & 0.421 & $\begin{array}{l}0.413(0.048- \\
3.550)\end{array}$ & & \\
\hline Unplanned pregnancy & 0.841 & $\begin{array}{l}1.149(0.296- \\
4.466)\end{array}$ & & \\
\hline $\begin{array}{l}\text { Method of diabetes control (diet vs. } \\
\text { medication) }\end{array}$ & 0.047 & $\begin{array}{l}4.125(1.017- \\
16.729)\end{array}$ & 0.029 & $\begin{array}{l}5.277(1.181- \\
23.580)\end{array}$ \\
\hline
\end{tabular}

Hosmer and Lemeshow goodness of fit (GOF) test; $\chi^{2}=9.812, \mathrm{df}=8, \mathrm{p}=0.278$. Cox and Snell pseudo$\mathrm{R}^{2}=0.045$. Nagelkerke pseudo- $\mathrm{R}^{2}=0.153$. 


\begin{tabular}{|c|c|c|c|}
\hline \multirow{2}{*}{$\begin{array}{l}\text { Variable } \\
\text { Maternal blood glucose control during } \\
\text { pregnancy }\end{array}$} & \multicolumn{2}{|c|}{$\begin{array}{l}\text { Univariate analysis }(n= \\
263)\end{array}$} & \multirow[t]{2}{*}{$\begin{array}{l}\text { Multivariate analysis }(n= \\
263)\end{array}$} \\
\hline & 0.838 & $\begin{array}{l}0.849(0.177- \\
4.077)\end{array}$ & \\
\hline $\begin{array}{l}\text { Mode of delivery (Cesarean vs. vaginal } \\
\text { birth) }\end{array}$ & & NA & \\
\hline Neonatal weight $\geq 3500 \mathrm{~g}$ & 0.865 & $\begin{array}{l}1.125(0.290- \\
4.371)\end{array}$ & \\
\hline \multicolumn{4}{|l|}{ Duration of labor (min) } \\
\hline Stage 1 & 0.076 & $\begin{array}{l}1.001(1.000- \\
1.003)\end{array}$ & \\
\hline Stage 2 & 0.583 & $\begin{array}{l}0.995(0.976- \\
1.014)\end{array}$ & \\
\hline Stage 3 & 0.581 & $\begin{array}{l}1.024(0.942- \\
1.113)\end{array}$ & \\
\hline
\end{tabular}

We then repeated the univariate and multivariate analyses for factors influencing the rate of neonate transfer to the NICU for hypoglycemia treatment after propensity score matching, using the same independent variables and parameters as described above (Table 9). From this multivariate analysis, only epidural analgesia was identified as a risk factor for neonatal hypoglycemia $(\mathrm{OR}, 12.526,95 \% \mathrm{Cl}, 1.322-$ 117.776; $p<0.05)$. 
Table 9

Results of univariate and multivariate analyses of factors influencing the rate of neonate transfer to the NICU for hypoglycemia treatment after propensity score matching

\begin{tabular}{|c|c|c|c|c|}
\hline \multirow{2}{*}{$\begin{array}{l}\text { Variable } \\
\text { Independent }\end{array}$} & \multicolumn{2}{|c|}{$\begin{array}{l}\text { Univariate analysis }(n= \\
\text { 138) }\end{array}$} & \multicolumn{2}{|c|}{$\begin{array}{l}\text { Multivariate analysis }(n= \\
138)\end{array}$} \\
\hline & $\mathbf{p}$ & OR $(95 \% \mathrm{Cl})$ & $\mathbf{p}$ & OR $(95 \% \mathrm{Cl})$ \\
\hline Epidural analgesia & 0.030 & $\begin{array}{l}10.200(1.255- \\
82.875)\end{array}$ & 0.027 & $\begin{array}{l}12.526(1.332- \\
117.776)\end{array}$ \\
\hline \multicolumn{5}{|l|}{ General information } \\
\hline Age (years) & 0.256 & $\begin{array}{l}0.903(0.757- \\
1.077)\end{array}$ & 0.160 & $\begin{array}{l}0.873(0.722- \\
1.055)\end{array}$ \\
\hline Gestational age at delivery (weeks) & 0.128 & $\begin{array}{l}1.729(0.855- \\
3.494)\end{array}$ & & \\
\hline $\mathrm{BMI}\left(\mathrm{kg} / \mathrm{m}^{2}\right)$ & 0.188 & $\begin{array}{l}1.106(0.952- \\
1.284)\end{array}$ & 0.104 & $\begin{array}{l}1.202(0.963- \\
1.501)\end{array}$ \\
\hline History of abnormal pregnancy & 0.520 & $\begin{array}{l}0.592(0.120- \\
2.919)\end{array}$ & & \\
\hline Pregnancy with obstetric disease & 0.948 & $\begin{array}{l}1.074(0.125- \\
9.219)\end{array}$ & & \\
\hline $\begin{array}{l}\text { Anxiety and depression during } \\
\text { pregnancy }\end{array}$ & 0.561 & $\begin{array}{l}1.921(0.212- \\
17.369)\end{array}$ & & \\
\hline History of depression and trauma & & NA & & \\
\hline $\begin{array}{l}\text { Cigarette, alcohol, and long-term } \\
\text { medication use }\end{array}$ & & NA & & \\
\hline \multicolumn{5}{|l|}{$\begin{array}{l}\text { Source of health knowledge during } \\
\text { pregnancy }\end{array}$} \\
\hline Routine obstetric examination & 0.204 & $\begin{array}{l}0.216(0.020- \\
2.292)\end{array}$ & & \\
\hline Maternity classes & 0.139 & $\begin{array}{l}0.364(0.095- \\
1.388)\end{array}$ & & \\
\hline Internet resources or books & & NA & & \\
\hline Unplanned pregnancy & 0.804 & $\begin{array}{l}0.817(0.164- \\
4.055)\end{array}$ & & \\
\hline $\begin{array}{l}\text { Method of diabetes control (diet vs. } \\
\text { medication) }\end{array}$ & 0.556 & $\begin{array}{l}0.613(0.120- \\
3.131)\end{array}$ & & \\
\hline
\end{tabular}

Data were matched by using propensity score matching with 1:1 nearest neighbor matching. Hosmer and Lemeshow goodness of fit (GOF) test; $\chi^{2}=9.992$, $d f=8, p=0.266$. McFadden's pseudo- $R^{2}=0.229$. Cox and Snell pseudo- $R^{2}=0.109$. Nagelkerke pseudo- $R^{2}=0.269$. 


\begin{tabular}{|c|c|c|c|c|}
\hline \multirow{2}{*}{$\begin{array}{l}\text { Variable } \\
\text { Maternal blood glucose control during } \\
\text { pregnancy }\end{array}$} & \multicolumn{2}{|c|}{$\begin{array}{l}\text { Univariate analysis }(n= \\
138)\end{array}$} & \multicolumn{2}{|c|}{$\begin{array}{l}\text { Multivariate analysis }(n= \\
\text { 138) }\end{array}$} \\
\hline & 0.017 & $\begin{array}{l}6.429(1.392- \\
29.694)\end{array}$ & 0.053 & $\begin{array}{l}5.384(0.976- \\
29.714)\end{array}$ \\
\hline $\begin{array}{l}\text { Mode of delivery (Cesarean vs. vaginal } \\
\text { birth) }\end{array}$ & & NA & & \\
\hline Neonatal weight $\geq 3500 \mathrm{~g}$ & 0.856 & $\begin{array}{l}1.139(0.279- \\
4.652)\end{array}$ & & \\
\hline \multicolumn{5}{|l|}{ Duration of labor (min) } \\
\hline Stage 1 & 0.053 & $\begin{array}{l}1.002(1.000- \\
1.003)\end{array}$ & & \\
\hline Stage 2 & 0.638 & $\begin{array}{l}0.996(0.977- \\
1.014)\end{array}$ & & \\
\hline Stage 3 & 0.352 & $\begin{array}{l}1.045(0.953- \\
1.146)\end{array}$ & & \\
\hline \multicolumn{5}{|c|}{$\begin{array}{l}\text { Data were matched by using propensity score matching with } 1: 1 \text { nearest neighbor matching. Hosmer } \\
\text { and Lemeshow goodness of fit }(G O F) \text { test; } \chi^{2}=9.992, d f=8, p=0.266 . \text { McFadden's pseudo- } R^{2}=0.229 \\
\text { Cox and Snell pseudo- } R^{2}=0.109 . \text { Nagelkerke pseudo- } R^{2}=0.269 .\end{array}$} \\
\hline
\end{tabular}

Thus, each analysis, with or without propensity score matching, identified one independent factor influencing the rate of neonate transfer to the NICU for hypoglycemia treatment was identified among parturients with GDM, but the factors differed with and without propensity score matching (method of diabetes control without propensity score matching and epidural labor analgesia with propensity score matching; Fig. 6).

Model for predicting neonate transfer to the NICU for hypoglycemia treatment after delivery by parturients with GDM

Two models based on the results of multiple logistic regression analysis were tested for their ability to predict the rate of neonate transfer to the NICU for hypoglycemia treatment. The model without propensity score matching showed an AUC value of 0.749 (95\% Cl: $0.567-0.930)$, with a sensitivity of 0.545 , specificity of 0.925 , positive predictive value of 0.240 , and negative predictive value of 0.979 . The model with propensity score matching showed an AUC value of 0.822 (95\% Cl: $0.672-0.972)$, with a sensitivity of 0.700 , specificity of 0.852 , positive predictive value of 0.240 , and negative predictive value of 0.965 (Fig. 7).

\section{Discussion}

The causes of PPD are complex, with many possible influencing factors [44, 45]. Early labor pain intensity is known to be associated with emotional disorder, and early postpartum labor pain is associated with 
PPD $[26,27]$. Furthermore, severe pain during delivery may be related to the patient's psychological reaction, and the influence of emotional state on pain experience has been widely recognized [46]. An adverse psychological state of parturients is closely related to the occurrence of PPD, and labor pain is recognized as an important cause of adverse emotions during childbirth [30]. In the present study, labor pain was assessed at three time points, and as expected, the pain scores (NRS scores) of patients who received epidural analgesia were significantly lower than those in the control group (Table 2). Several studies have reported that epidural labor analgesia may decrease the incidence of PPD $[16,26,28,30]$, with two of these studies finding a positive correlation between labor pain score and the early postpartum EPDS score [26, 30]. Boudou et al [26] reported a significant positive correlation between labor pain and the EPDS score specifically at 3 days postpartum, while Hiltunen et al [30] reported that pain relief during vaginal delivery decreased the risk of PPD, especially immediately after delivery. Ding et al [16] reported that epidural labor analgesia decreased the incidence of PPD at 42 days after delivery. The increased emotional response to anticipated pain is known to impair the ability to regulate pain, and the brain neural network activated during the experience of psychological pain overlaps with the brain regions involved in physical pain [47]. In parturients suffering from pain and/or major depression, the ability to respond to emotional information is impaired [48]. However, other studies found no relationship between epidural analgesia and PPD [9, 12, 49-52], and currently, the American College of Obstetrics and Gynecology (ACOG) and the United States Preventive Services Task Force do not regard labor pain as a risk factor for PPD [42, 51]. In addition to labor pain, other factors that have been found to be independently associated with PPD include: prenatal depression, self-esteem, childcare stress, prenatal anxiety, life stress, social support, marital relationship, history of previous depression, infant temperament, maternity blues, marital status, socioeconomic status, and unplanned/unwanted pregnancy [53]. Thus, additional studies are needed to clarify the relationship between epidural labor analgesia and PPD in the general population of pregnant mothers and in those with specific conditions, such as GDM.

Previous research has also implicated GDM as a potential risk factor of PPD. In a study of 1801 women in Iran, Abdollahi et al [54] found a significant correlation between GDM and PPD (OR $=2.93,95 \% \mathrm{Cl}$, 1.46-5.88). The United States Preventive Services Task Force also lists GDM as a risk factor for PPD [55]. GDM creates a background of changes in metabolic status, the inflammatory state, and the hypothalamus-pituitary-adrenal axis that are associated with an increased risk of depression.[34-36] However, in the study of Miller et al [56], the relationship between GDM and PPD was not significant. The reported rates of PPD in the general maternal population are within the range of $11-19.2 \%(13 \%)[13,14]$, and in the parturients with GDM in the present study, the incidence rates of PPD (11.28-18.8\%) were not higher than this normal range. However, our multivariate analysis after propensity score matching for variable that differed between our epidural and control groups did identify epidural labor analgesia as a protective factor for PPD at $24 \mathrm{~h}$ post-delivery (OR, $0.301,95 \% \mathrm{Cl}: 0.104-0.867 ; \mathrm{p}<0.05)$. In our analysis, the significant independent variables were age, gravidity, history of abnormal pregnancy, occupation, and SSRS. We applied these variables as independent variables, used the 1:1 nearest neighbor matching 
method, and conducted the logistic regression analysis after propensity score matching to reduce potential selection bias.

In the present study, SAS score and unplanned pregnancy were also found to be risk factors of PPD at 24 $\mathrm{h}$ after delivery in parturients with GDM, while anxiety and depression during pregnancy and unplanned pregnancy were risk factors for PPD at 42 days post-delivery in our patients. It is well known that the physiological and psychological changes during pregnancy are likely to induce anxiety and other emotional problems during childbirth. Anxiety is a normal cognitive activity and emotional response, but excessive anxiety can increase the risk of mental illness [57]. A retrospective study of 2926 male and 1929 female adult twins [58] showed that anxiety is the primary risk factor for major depressive disorder (MDD). Previous research has also found that perinatal anxiety and depression are important risk factors of PPD [59-61]. Heron et al [62] suggested that antenatal anxiety might predict PPD at 8 weeks and 8 months after delivery. The SAS is commonly used to evaluate the anxiety state of parturients before and after delivery, as it reflects internalized factors [63], and the SSRS is often used to assess women's social support [64]. In the present study, a high SAS score was a risk factor for PPD, and a high SSRS score was a protective factor against PPD, which is consistent with conclusions in the existing literature $[53,65]$. Positive social support is one of the most effective means to overcoming stress and has been correlated with the relief of depression and anxiety disorder [66].

Unplanned pregnancy has also been shown to be a risk factor for PPD in general populations,[67-69] and studies have found that approximately half of pregnancies are unplanned. $[65,66]$ A multicenter study of 290 Japanese women [67] showed that women with an unplanned pregnancy had a higher incidence of severe depression during pregnancy and of PPD at 3 months after delivery. Another study [68] of 2076 South Korean women showed that the risk of depression was increased by $20-22 \%$ for an unplanned pregnancy. Another prospective study of 688 women in the United States found that unplanned pregnancy was a risk factor for PPD at 3 and 12 months after delivery [69]. In the present study of parturients with GDM, our results indicate that unplanned pregnancy was a risk factor for PPD at $24 \mathrm{~h}$ and 42 days after delivery, consistent with the literature described above [67-69]. These findings have important implications for future research and women's pregnancy care, as women with unplanned pregnancy should be regarded as an at-risk population for early detection and prevention of PPD [70].

In the present study, epidural labor analgesia significantly prolonged the first and second stages of labor, but the durations of these stages were still within the normal ranges (Table 2), as experts have concluded that the upper limit for the second stage delivery should be $4 \mathrm{~h}$ for primiparas with epidural analgesia and $3 \mathrm{~h}$ for primiparas without epidural analgesia [71]. Previous studies have also reported that epidural labor analgesia can prolong the first and second stages of labor [22, 72-74], which is consistent with our results. Inconsistently though, two studies $[22,75]$ reported that epidural administration of $0.08 \%$ ropivacaine and $0.4 \mu \mathrm{g} / \mathrm{ml}$ sufentanil did not prolong the second stage of labor. This discrepancy may be related to the use of different local anesthetic concentrations ( $0.1 \%$ ropivacaine and $0.4 \mu \mathrm{g} / \mathrm{ml}$ sufentanil in the present study). In this study, the incidence rates of pruritus, dizziness, and urine retention were higher in the epidural group than in the control group, which may be related to the epidural administration 
of opioids [39, 41]. Every treatment has advantages and disadvantages, and indeed, epidural analgesia can induce side effects while reducing labor pain. Importantly, in this study, epidural labor analgesia did not increase the percentage of patients who required delivery by cesarean section. The vaginal delivery rate in the epidural group was still similar to that in the control group, which is encouraging. Thus, although epidural analgesia may have prolonged the first and second stages of labor and induced mild side effects, the delivery outcome was not affected. Furthermore, from our logistic regression analysis, the duration of the labor stage was not associated with an increased risk of PPD.

Diabetes control with medication and epidural analgesia were both identified as risk factors for neonate transfer to the NICU for hypoglycemia treatment among our GDM population. In women with GDM, diet is the first choice for blood glucose control, but if the effect is insufficient, drug control is the better choice. This means that women who require drug control are more likely to have experienced poor blood glucose control. Blachier et al [76] found that neonatal hypoglycemia is associated with drug control of GDM and not associated with diet control of GDM, which is consistent with our results.

The pain and tension experienced during the perinatal period lead to a significant increase in the blood glucose concentration [77], and the fetal plasma catecholamine level can reach a very high level during delivery. This increase can be avoided by appropriate maternal pain relief and sympathetic block after epidural anesthesia [78]. Indeed, epidural anesthesia reduces maternal stress hormone levels during labor [8]. Studies have found that $6 \mathrm{~h}$ after delivery, the plasma cortisol level of parturients who received epidural anesthesia is lower than that in those who did not receive epidural anesthesia [9]. This study found that the incidence of hypoglycemia in newborns at $2 \mathrm{~h}$ and $3 \mathrm{~h}$ after birth as well as the proportion of neonates with hypoglycemia requiring NICU treatment were higher in the epidural group than in the control group. These differences may be due to the fact that labor analgesia inhibits the stress response induced by pain and tension. If the concentration of catecholamine is significantly reduced, the increase in blood glucose concentration may also be inhibited [79]. Another study showed that during childbirth, the serum concentrations of cortisol and adrenaline of mothers abruptly decreased, which could trigger an acute hypoglycemic event [80]. With the onset of pain relief, the catecholamine concentration decreases significantly, potentially resulting in an acute hypoglycemia attack in a diabetic parturient after administration of labor analgesia [37]. Our results showed that the incidence of postpartum newborn hypoglycemia ( $<47 \mathrm{mg} / \mathrm{dl}[2.6 \mathrm{mmol} / \mathrm{l}]$ ) was $11.54 \%$ in the epidural group and $6.02 \%$ in the control group. A cohort study in an Israeli medical center reported an incidence of neonatal hypoglycemia of $12.1 \%$ among all newborns at 74 min after delivery [81]. The lower rates in our study population may be due to differences in the subjects and deliveries. Overall, epidural analgesia is known to potentially lead to maternal hypoglycemia, which then leads to neonatal hypoglycemia. Because repeated or persistent severe hypoglycemia may cause damage to the central nervous system [75], it is necessary to monitor neonatal blood glucose levels closely after administration of epidural analgesia and apply timely treatment for neonatal hypoglycemia.

The optimal timing of PPD assessment has been controversial. One study found that when commonly used depression rating scales were administered to identify PPD immediately after delivery, their 
psychometric properties were not satisfactory [82]. Although depression symptoms immediately after delivery are frequent but transient, application of the EPDS subscale immediately after delivery provides insight into a spectrum of postpartum psychological problems, which are associated with later depressive disorders [40]. In the present study, the EPDS score was assessed at $24 \mathrm{~h}$ after delivery and then again at 42 days after delivery. The EPDS score at 42 days was used for preliminary screening for diagnosis of PPD. Our analysis did reveal a significant correlation between the EPDS score at $24 \mathrm{~h}$ postdelivery and that at 42 days after delivery (Pearson correlation coefficient $=0.527, p<0.001$ ).

This study has several limitations that should be considered. First, continuous monitoring of the blood glucose concentration of parturients was lacking, as invasive blood glucose monitoring was rejected by the patients and not strictly enforced. Second, group allocation was determined according to maternal choice. Third, the presence of depression was not assessed before delivery. Fourth, PPD was not diagnosed by psychiatrists; however, the EPDS is an established tool for the detection of PPD by nonpsychiatrists [83, 84] and has been well verified in China [85]. Lastly, the follow-up time was short at only 42 days.

Several advantages of the present study are also worth noting. To our knowledge, this was the first study to investigate the effects of epidural analgesia in pregnant women with GDM and their newborns. The sample size was 263 , which is larger than the samples sizes of relevant previous studies $[16,86]$. We also retained $86.5 \%$ of the study participants at the final follow-up. Although we found that epidural analgesia could prolong the first and second stages of labor and increase the incidence rates of maternal side effects and neonatal hypoglycemia, its clinical benefit (reducing labor pain) was still clear. More research is needed to understand the association between epidural analgesia and hypoglycemia of neonates born to parturients with GDM, as this study identifies such an association for the first time. Additionally, more research on the optimal drug combination and dosage for epidural labor analgesia is needed in the future to improve pain relief while reducing the incidence of side effects.

Epidural labor analgesia may be a protective factor against PPD but a risk factor for neonatal hypoglycemia. Further studies with a larger sample size and longer follow-up time are needed to better characterize the effect of epidural labor analgesia on PPD and neonatal hypoglycemia.

\section{Conclusion}

Epidural labor analgesia may be a protective factor against PPD but a risk factor for neonatal hypoglycemia. Further studies with a larger sample size and longer follow-up time are needed to better characterize the effect of epidural labor analgesia on PPD and neonatal hypoglycemia.

\section{Abbreviations}

PPD, Postpartum depression; GDM, Gestational diabetes mellitus; EPDS, Edinburgh Postnatal Depression Scale; BMI, Body mass index; OGTT, Oral glucose tolerance test; NRS, Numerical Rating Scale; 
PCEA, Patient-controlled epidural analgesia; NICU, Neonatal intensive care unit; SAS, Zung Self-Rating Anxiety Scale;

SSRS, Social Support Rating Scale.

\section{Declarations}

\section{Ethics approval and consent to participate}

Written informed consent was obtained from all enrolled parturients. The study was conducted in accordance with the Declaration of Helsinki and Chinese clinical trial research regulations. The study protocol was approved by the Shenzhen Maternity and Child Healthcare Hospital Ethics Committee (Approval No. SZFY2017102095). and the study was registered in the China Clinical Registration Center (Registration No. ChiCTR-O0C-17013164). Written informed consent was obtained from all participants.

\section{Consent for publication}

All participants have given consent for publication.

\section{Availability of data and materials}

All data generated or analyzed during this study are included in this published article.

\section{Competing interest}

We declare that we have no conflict of interest.

\section{Funding}

No funds, grants, or other support was received.

\section{Authors' contributions}

$\mathrm{GHL}, \mathrm{XFQ}$ contributed to the design of the study, data analysis and interpretation, manuscript drafting and review. XHT, MGW, HW, XGW, JS, and YL contributed to the design of the study, data analysis, data interpretation and review of manuscript to be published. YTL, XLH and PWcontributed to the conception and design of the study, and review of manuscript to be published.

\section{Acknowledgments}

Not applicable.

\section{References}


[1] Oats JJ (1998) Fourth International Workshop-Conference on Gestational Diabetes Mellitus. Overview and commentary on first session. Diabetes Care 21 Suppl 2:B58-59

[2] Zhu Y, Zhang C (2016) Prevalence of Gestational Diabetes and Risk of Progression to Type 2 Diabetes: a Global Perspective. Curr Diab Rep 16 (1):7. doi:10.1007/s11892-015-0699-x

[3] Mitanchez D (2010) [Fetal and neonatal complications of gestational diabetes: perinatal mortality, congenital malformations, macrosomia, shoulder dystocia, birth injuries, neonatal outcomes]. J Gynecol Obstet Biol Reprod (Paris) 39 (8 Suppl 2):S189-199. doi:10.1016/s0368-2315(10)70046-6

[4] Ostlund I, Hanson U, Björklund A, Hjertberg R, Eva N, Nordlander E, Swahn ML, Wager J (2003) Maternal and fetal outcomes if gestational impaired glucose tolerance is not treated. Diabetes Care 26 (7):2107-2111. doi:10.2337/diacare.26.7.2107

[5] Hedderson MM, Ferrara A, Sacks DA (2003) Gestational diabetes mellitus and lesser degrees of pregnancy hyperglycemia: association with increased risk of spontaneous preterm birth. Obstet Gynecol 102 (4):850-856. doi:10.1016/s0029-7844(03)00661-6

[6] Metzger BE, Persson B, Lowe LP, Dyer AR, Cruickshank JK, Deerochanawong C, Halliday HL, Hennis AJ, Liley H, Ng PC, Coustan DR, Hadden DR, Hod M, Oats JJ, Trimble ER (2010) Hyperglycemia and adverse pregnancy outcome study: neonatal glycemia. Pediatrics 126 (6):e1545-1552. doi:10.1542/peds.20092257

[7] Farahvar S, Walfisch A (2019) Gestational diabetes risk factors and long-term consequences for both mother and offspring: a literature review. Expert Rev Endocrinol Metab 14 (1):63-74.

doi:10.1080/17446651.2018.1476135

[8] Westgren M, Lindahl SG, Nordén NE (1986) Maternal and fetal endocrine stress response at vaginal delivery with and without an epidural block. J Perinat Med 14 (4):235-241.

doi:10.1515/jpme.1986.14.4.235

[9] Riazanova OV, Alexandrovich YS, loscovich AM (2018) The relationship between labor pain management, cortisol level and risk of postpartum depression development: a prospective nonrandomized observational monocentric trial. Rom J Anaesth Intensive Care 25 (2):123-130. doi:10.21454/rjaic.7518.252.rzn

[10] Gaynes BN, Gavin N, Meltzer-Brody S, Lohr KN, Swinson T, Gartlehner G, Brody S, Miller WC (2005) Perinatal depression: prevalence, screening accuracy, and screening outcomes. Evid Rep Technol Assess (Summ) (119):1-8. doi:10.1037/e439372005-001

[11] O'Hara MW, Swain AM (1996) Rates and risk of postpartum depression-a meta-analysis. Int Rev Psychiatry 8:37-54 
[12] Nahirney M, Metcalfe A, Chaput KH (2017) Administration of epidural labor analgesia is not associated with a decreased risk of postpartum depression in an urban Canadian population of mothers: a secondary analysis of prospective cohort data. Local Reg Anesth 10:99-104. doi:10.2147/Ira.s141569

[13] Gavin NI, Gaynes BN, Lohr KN, Meltzer-Brody S, Gartlehner G, Swinson T (2005) Perinatal depression: a systematic review of prevalence and incidence. Obstet Gynecol 106 (5 Pt 1):1071-1083.

doi:10.1097/01.AOG.0000183597.31630.db

[14] Ko JY, Rockhill KM, Tong VT, Morrow B, Farr SL (2017) Trends in Postpartum Depressive Symptoms 27 States, 2004, 2008, and 2012. MMWR Morb Mortal Wkly Rep 66 (6):153-158. doi:10.15585/mmwr.mm6606a1

[15] Woody CA, Ferrari AJ, Siskind DJ, Whiteford HA, Harris MG (2017) A systematic review and metaregression of the prevalence and incidence of perinatal depression. J Affect Disord 219:86-92. doi:10.1016/j.jad.2017.05.003

[16] Ding T, Wang DX, Qu Y, Chen Q, Zhu SN (2014) Epidural labor analgesia is associated with a decreased risk of postpartum depression: a prospective cohort study. Anesth Analg 119 (2):383-392. doi:10.1213/ane.0000000000000107

[17] Liu S, Yan Y (2017) Risk factors for postpartum depression among Chinese women: path model analysis. BMC Pregnancy Childbirth 17 (1):133. doi:10.1186/s12884-017-1320-x

[18] Wang YY, Li H, Wang YJ, Wang H, Zhang YR, Gong L, Ma J, Wang Y, Wang MZ, Qiu SX, Yuan SX (2017) Living with parents or with parents-in-law and postpartum depression: A preliminary investigation in China. J Affect Disord 218:335-338. doi:10.1016/j.jad.2017.04.052

[19] Xie RH, He G, Liu A, Bradwejn J, Walker M, Wen SW (2007) Fetal gender and postpartum depression in a cohort of Chinese women. Soc Sci Med 65 (4):680-684. doi:10.1016/j.socscimed.2007.04.003

[20] Dennis CL, McQueen K (2009) The relationship between infant-feeding outcomes and postpartum depression: a qualitative systematic review. Pediatrics 123 (4):e736-751. doi:10.1542/peds.2008-1629

[21] Brummelte S, Galea LA (2016) Postpartum depression: Etiology, treatment and consequences for maternal care. Horm Behav 77:153-166. doi:10.1016/j.yhbeh.2015.08.008

[22] Murray L, Cooper P (1997) Effects of postnatal depression on infant development. Arch Dis Child 77 (2):99-101. doi:10.1136/adc.77.2.99

[23] Hoffman C, Dunn DM, Njoroge WFM (2017) Impact of Postpartum Mental Illness Upon Infant Development. Curr Psychiatry Rep 19 (12):100. doi:10.1007/s11920-017-0857-8

[24] Farías-Antúnez S, Xavier MO, Santos IS (2018) Effect of maternal postpartum depression on offspring's growth. J Affect Disord 228:143-152. doi:10.1016/j.jad.2017.12.013 
[25] Kingston D, Tough S, Whitfield H (2012) Prenatal and postpartum maternal psychological distress and infant development: a systematic review. Child Psychiatry Hum Dev 43 (5):683-714.

doi:10.1007/s10578-012-0291-4

[26] Boudou $M$, Teissèdre F, Walburg V, Chabrol H (2007) Association between the intensity of childbirth pain and the intensity of postpartum blues. Encephale 33 (5):805-810. doi:10.1016/j.encep.2006.10.002

[27] Eisenach JC, Pan PH, Smiley R, Lavand'homme P, Landau R, Houle TT (2008) Severity of acute pain after childbirth, but not type of delivery, predicts persistent pain and postpartum depression. Pain 140 (1):87-94. doi:10.1016/j.pain.2008.07.011

[28] Suhitharan T, Pham TP, Chen H, Assam PN, Sultana R, Han NL, Tan EC, Sng BL (2016) Investigating analgesic and psychological factors associated with risk of postpartum depression development: a casecontrol study. Neuropsychiatr Dis Treat 12:1333-1339. doi:10.2147/ndt.s105918

[29] Orbach-Zinger S, Landau R, Harousch AB, Ovad O, Caspi L, Kornilov E, loscovich A, Bracco D, Davis A, Fireman S, Hoshen M, Eidelman LA (2018) The Relationship Between Women's Intention to Request a Labor Epidural Analgesia, Actually Delivering With Labor Epidural Analgesia, and Postpartum Depression at 6 Weeks: A Prospective Observational Study. Anesth Analg 126 (5):1590-1597. doi:10.1213/ane.0000000000002501

[30] Hiltunen P, Raudaskoski T, Ebeling H, Moilanen I (2004) Does pain relief during delivery decrease the risk of postnatal depression? Acta Obstet Gynecol Scand 83 (3):257-261. doi:10.1111/j.00016349.2004.0302.x

[31] Silverman ME, Reichenberg A, Savitz DA, Cnattingius S, Lichtenstein P, Hultman CM, Larsson H, Sandin S (2017) The risk factors for postpartum depression: A population-based study. Depress Anxiety 34 (2):178-187. doi:10.1002/da.22597

[32] Azami M, Badfar G, Soleymani A, Rahmati S (2019) The association between gestational diabetes and postpartum depression: A systematic review and meta-analysis. Diabetes Res Clin Pract 149:147155. doi:10.1016/j.diabres.2019.01.034

[33] Ruohomäki A, Toffol E, Upadhyaya S, Keski-Nisula L, Pekkanen J, Lampi J, Voutilainen S, Tuomainen TP, Heinonen S, Kumpulainen K, Pasanen M, Lehto SM (2018) The association between gestational diabetes mellitus and postpartum depressive symptomatology: A prospective cohort study. J Affect Disord 241:263-268. doi:10.1016/j.jad.2018.08.070

[34] Hinkle SN, Buck Louis GM, Rawal S, Zhu Y, Albert PS, Zhang C (2016) A longitudinal study of depression and gestational diabetes in pregnancy and the postpartum period. Diabetologia 59 (12):25942602. doi:10.1007/s00125-016-4086-1 
[35] Sandu RE, Buga AM, Uzoni A, Petcu EB, Popa-Wagner A (2015) Neuroinflammation and comorbidities are frequently ignored factors in CNS pathology. Neural Regen Res 10 (9):1349-1355. doi:10.4103/16735374.165208

[36] Huang T, Rifas-Shiman SL, Ertel KA, Rich-Edwards J, Kleinman K, Gillman MW, Oken E, James-Todd T (2015) Pregnancy Hyperglycaemia and Risk of Prenatal and Postpartum Depressive Symptoms. Paediatr Perinat Epidemiol 29 (4):281-289. doi:10.1111/ppe.12199

[37] Metzger BE, Gabbe SG, Persson B, Buchanan TA, Catalano PA, Damm P, Dyer AR, Leiva A, Hod M, Kitzmiler JL, Lowe LP, McIntyre HD, Oats JJ, Omori Y, Schmidt MI (2010) International association of diabetes and pregnancy study groups recommendations on the diagnosis and classification of hyperglycemia in pregnancy. Diabetes Care 33 (3):676-682. doi:10.2337/dc09-1848

[38] Thong ISK, Jensen MP, Miró J, Tan G (2018) The validity of pain intensity measures: what do the NRS, VAS, VRS, and FPS-R measure? Scand J Pain 18 (1):99-107. doi:10.1515/sjpain-2018-0012

[39] Fugelseth D (2001) [Neonatal hypoglycemia]. Tidsskr Nor Laegeforen 121 (14):1713-1716

[40] Petrozzi A, Gagliardi L (2013) Anxious and depressive components of Edinburgh Postnatal Depression Scale in maternal postpartum psychological problems. J Perinat Med 41 (4):343-348. doi:10.1515/jpm-2012-0258

[41] Cox JL, Holden JM, Sagovsky R (1987) Detection of postnatal depression. Development of the 10item Edinburgh Postnatal Depression Scale. Br J Psychiatry 150:782-786. doi:10.1192/bjp.150.6.782

[42] Zung WW (1971) A rating instrument for anxiety disorders. Psychosomatics 12 (6):371-379. doi:10.1016/s0033-3182(71)71479-0

[43] Xiao S (1994) Theoretical basis and application in research of Social Support Rating Scale. J Clin Psychiatry 4

[44] Yim IS, Tanner Stapleton LR, Guardino CM, Hahn-Holbrook J, Dunkel Schetter C (2015) Biological and psychosocial predictors of postpartum depression: systematic review and call for integration. Annu Rev Clin Psychol 11:99-137. doi:10.1146/annurev-clinpsy-101414-020426

[45] Šebela A, Hanka J, Mohr P (2018) Etiology, risk factors, and methods of postpartum depression prevention. Ceska Gynekol 83 (6):468-473

[46] Wisner KL, Stika CS, Clark CT (2014) Double duty: does epidural labor analgesia reduce both pain and postpartum depression? Anesth Analg 119 (2):219-221. doi:10.1213/ane.0000000000000322

[47] Meerwijk EL, Ford JM, Weiss SJ (2013) Brain regions associated with psychological pain: implications for a neural network and its relationship to physical pain. Brain Imaging Behav 7 (1):1-14. doi:10.1007/s11682-012-9179-y 
[48] Strigo IA, Matthews SC, Simmons AN (2013) Decreased frontal regulation during pain anticipation in unmedicated subjects with major depressive disorder. Transl Psychiatry 3 (3):e239.

doi:10.1038/tp.2013.15

[49] Tobin CD, Wilson SH, Hebbar L, Roberts LL, Wolf BJ, Guille C (2017) Labor Epidural Analgesia and Postpartum Depression. Arch Depress Anxiety 2 (2):044-046. doi:10.17352/2455-5460.000014

[50] Eckerdal P, Kollia N, Karlsson L, Skoog-Svanberg A, Wikström AK, Högberg U, Skalkidou A (2020) Epidural Analgesia During Childbirth and Postpartum Depressive Symptoms: A Population-Based Longitudinal Cohort Study. Anesth Analg 130 (3):615-624. doi:10.1213/ane.0000000000004292

[51] Kountanis JA, Vahabzadeh C, Bauer S, Muzik M, Cassidy R, Aman C, MacEachern M, Bauer ME (2020) Labor epidural analgesia and the risk of postpartum depression: A meta-analysis of observational studies. J Clin Anesth 61:109658. doi:10.1016/j.jclinane.2019.109658

[52] Almeida M, Kosman KA, Kendall MC, De Oliveira GS (2020) The association between labor epidural analgesia and postpartum depression: a systematic review and meta-analysis. BMC Womens Health 20 (1):99. doi:10.1186/s12905-020-00948-0

[53] Beck CT (2001) Predictors of postpartum depression: an update. Nurs Res 50 (5):275-285. doi:10.1097/00006199-200109000-00004

[54] Abdollahi F, Zarghami M, Azhar MZ, Sazlina SG, Lye MS (2014) Predictors and incidence of postpartum depression: a longitudinal cohort study. J Obstet Gynaecol Res 40 (12):2191-2200. doi:10.1111/jog.12471

[55] Curry SJ, Krist AH, Owens DK, Barry MJ, Caughey AB, Davidson KW, Doubeni CA, Epling JW, Jr., Grossman DC, Kemper AR, Kubik M, Landefeld CS, Mangione CM, Silverstein M, Simon MA, Tseng CW, Wong JB (2019) Interventions to Prevent Perinatal Depression: US Preventive Services Task Force Recommendation Statement. Jama 321 (6):580-587. doi:10.1001/jama.2019.0007

[56] Miller ES, Peri MR, Gossett DR (2016) The association between diabetes and postpartum depression. Arch Womens Ment Health 19 (1):183-186. doi:10.1007/s00737-015-0544-x

[57] Affonso DD, Liu-Chiang CY, Mayberry LJ (1999) Worry: conceptual dimensions and relevance to childbearing women. Health Care Women Int 20 (3):227-236. doi:10.1080/073993399245728

[58] Stewart DE, Vigod SN (2019) Postpartum Depression: Pathophysiology, Treatment, and Emerging Therapeutics. Annu Rev Med 70:183-196. doi:10.1146/annurev-med-041217-011106

[59] Hettema JM, Prescott CA, Kendler KS (2003) The effects of anxiety, substance use and conduct disorders on risk of major depressive disorder. Psychol Med 33 (8):1423-1432.

doi:10.1017/s0033291703008365 
[60] Alipour Z, Lamyian M, Hajizadeh E (2012) Anxiety and fear of childbirth as predictors of postnatal depression in nulliparous women. Women Birth 25 (3):e37-43. doi:10.1016/j.wombi.2011.09.002

[61] Austin MP, Tully L, Parker G (2007) Examining the relationship between antenatal anxiety and postnatal depression. J Affect Disord 101 (1-3):169-174. doi:10.1016/j.jad.2006.11.015

[62] Heron J, O'Connor TG, Evans J, Golding J, Glover V (2004) The course of anxiety and depression through pregnancy and the postpartum in a community sample. J Affect Disord 80 (1):65-73. doi:10.1016/j.jad.2003.08.004

[63] Liu X, Chen M, Wang Y, Sun L, Zhang J, Shi Y, Wang J, Zhang H, Sun G, Baker PN, Luo X, Qi H (2020) Prenatal anxiety and obstetric decisions among pregnant women in Wuhan and Chongqing during the COVID-19 outbreak: a cross-sectional study. Bjog 127 (10):1229-1240. doi:10.1111/1471-0528.16381

[64] O'Hara MW (2009) Postpartum depression: what we know. J Clin Psychol 65 (12):1258-1269. doi:10.1002/jclp.20644

[65] Li Y, Long Z, Cao D, Cao F (2017) Social support and depression across the perinatal period: A longitudinal study. J Clin Nurs 26 (17-18):2776-2783. doi:10.1111/jocn.13817

[66] Kim HS, Sherman DK, Taylor SE (2008) Culture and social support. Am Psychol 63 (6):518-526. doi:10.1037/0003-066x

[67] Kitamura T, Yoshida K, Okano T, Kinoshita K, Hayashi M, Toyoda N, Ito M, Kudo N, Tada K, Kanazawa K, Sakumoto K, Satoh S, Furukawa T, Nakano H (2006) Multicentre prospective study of perinatal depression in Japan: incidence and correlates of antenatal and postnatal depression. Arch Womens Ment Health 9 (3):121-130. doi:10.1007/s00737-006-0122-3

[68] Bahk J, Yun SC, Kim YM, Khang YH (2015) Impact of unintended pregnancy on maternal mental health: a causal analysis using follow up data of the Panel Study on Korean Children (PSKC). BMC Pregnancy Childbirth 15:85. doi:10.1186/s12884-015-0505-4

[69] Mercier RJ, Garrett J, Thorp J, Siega-Riz AM (2013) Pregnancy intention and postpartum depression: secondary data analysis from a prospective cohort. Bjog 120 (9):1116-1122. doi:10.1111/14710528.12255

[70] Baba S, Kimura T, Ikehara S, Honjo K, Eshak ES, Sato T, Iso H (2020) Impact of intention and feeling toward being pregnant on postpartum depression: the Japan Environment and Children's Study (JECS). Arch Womens Ment Health 23 (1):131-137. doi:10.1007/s00737-018-0938-7

[71] Leveno KJ, Nelson DB, Mclntire DD (2016) Second-stage labor: how long is too long? Am J Obstet Gynecol 214 (4):484-489. doi:10.1016/j.ajog.2015.10.926 
[72] Zhang J, Yancey M, Klebanoff M, Schwarz J, Schweitzer D (2000) Effects of epidural analgesia on the course of labor and delivery: a natural experiment. Obstetrics \& Gynecology 95 (4):S45

[73] Costa-Martins JM, Dias CC, Pereira M, Tavares J (2015) Effects of local anesthetic on the time between analgesic boluses and the duration of labor in patient-controlled epidural analgesia: prospective study of two ultra-low dose regimens of ropivacaine and sufentanil. Acta Med Port 28 (1):70-76. doi:10.20344/amp.5708

[74] Rimaitis K, Klimenko O, Rimaitis M, Morkūnaitè A, Macas A (2015) Labor epidural analgesia and the incidence of instrumental assisted delivery. Medicina (Kaunas) 51 (2):76-80.

doi:10.1016/j.medici.2015.02.002

[75] Kerstjens JM, Bocca-Tjeertes IF, de Winter AF, Reijneveld SA, Bos AF (2012) Neonatal morbidities and developmental delay in moderately preterm-born children. Pediatrics 130 (2):e265-272. doi:10.1542/peds.2012-0079

[76] Blachier A, Alberti C, Korb D, Schmitz T, Patrick V, Christine B, Oury JF, Sibony O (2014) Diet or medically treated gestational diabetes: is there any difference for obstetrical and neonatal complications? A French cohort study. J Perinat Med 42 (3):315-319. doi:10.1515/.jpm-2013-0079

[77] Glosten B (1993) Anesthesia and coexisting maternal disease. Part two: Diabetes mellitus, obesity, and pulmonary and neurology disease. In: Obstetric anesthesia. JB Lippincott Company Philadelphia, pp $473-450$

[78] Irestedt L, Lagercrantz H, Belfrage P (1984) Causes and consequences of maternal and fetal sympathoadrenal activation during parturition. Acta Obstet Gynecol Scand Suppl 118:111-115. doi:10.3109/00016348409157136

[79] Cascio M, Pygon B, Bernett C, Ramanathan S (1997) Labour analgesia with intrathecal fentanyl decreases maternal stress. Can J Anaesth 44 (6):605-609. doi:10.1007/bf03015443

[80] Crites J, Ramanathan J (2000) Acute hypoglycemia following combined spinal-epidural anesthesia (CSE) in a parturient with diabetes mellitus. Anesthesiology 93 (2):591-592. doi:10.1097/00000542200008000-00061

[81] Bromiker R, Perry A, Kasirer Y, Einav S, Klinger G, Levy-Khademi F (2019) Early neonatal hypoglycemia: incidence of and risk factors. A cohort study using universal point of care screening. $J$ Matern Fetal Neonatal Med 32 (5):786-792. doi:10.1080/14767058.2017.1391781

[82] Lee DT, Yip AS, Chan SS, Tsui MH, Wong WS, Chung TK (2003) Postdelivery screening for postpartum depression. Psychosom Med 65 (3):357-361. doi:10.1097/01.psy.0000035718.37593.87

[83] Beck CT, Gable RK (2001) Comparative analysis of the performance of the Postpartum Depression Screening Scale with two other depression instruments. Nurs Res 50 (4):242-250. doi:10.1097/00006199- 
[84] Lee DT, Yip AS, Chiu HF, Leung TY, Chung TK (2001) Screening for postnatal depression: are specific instruments mandatory? J Affect Disord 63 (1-3):233-238. doi:10.1016/s0165-0327(00)00193-2

[85] Lee DT, Yip SK, Chiu HF, Leung TY, Chan KP, Chau IO, Leung HC, Chung TK (1998) Detecting postnatal depression in Chinese women. Validation of the Chinese version of the Edinburgh Postnatal Depression Scale. Br J Psychiatry 172:433-437. doi:10.1192/bjp.172.5.433

[86] Lim G, Farrell LM, Facco FL, Gold MS, Wasan AD (2018) Labor Analgesia as a Predictor for Reduced Postpartum Depression Scores: A Retrospective Observational Study. Anesth Analg 126 (5):1598-1605. doi:10.1213/ane.0000000000002720

\section{Figures}




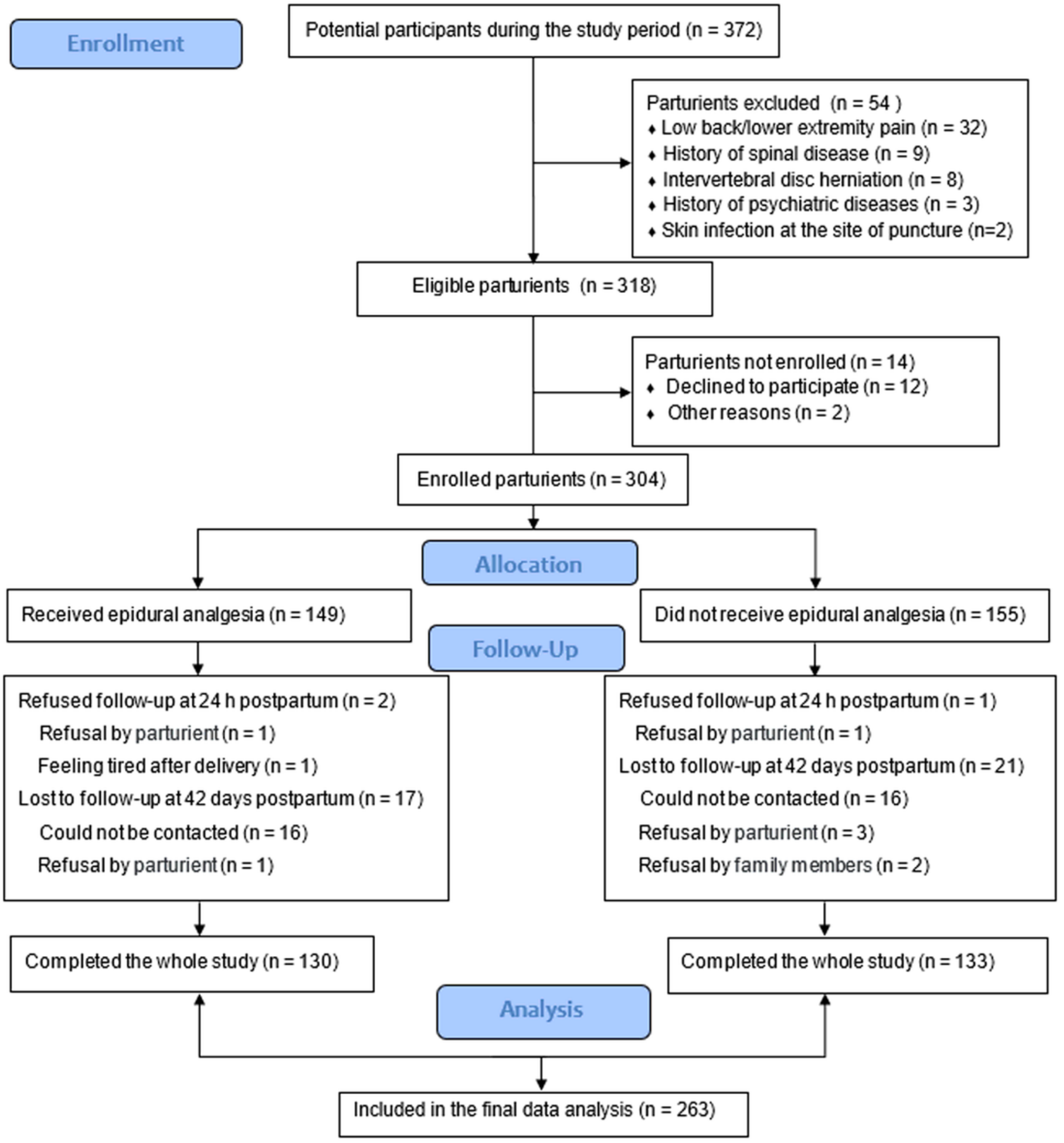

\section{Figure 1}

Flow chart of patient enrollment. 
Factors

Original data $(n=263)$

Epidural analgesia

SAS

SSRS

Matched by PSM $(n=138)$

Epidural analgesia

Unplanned pregnancy

SAS

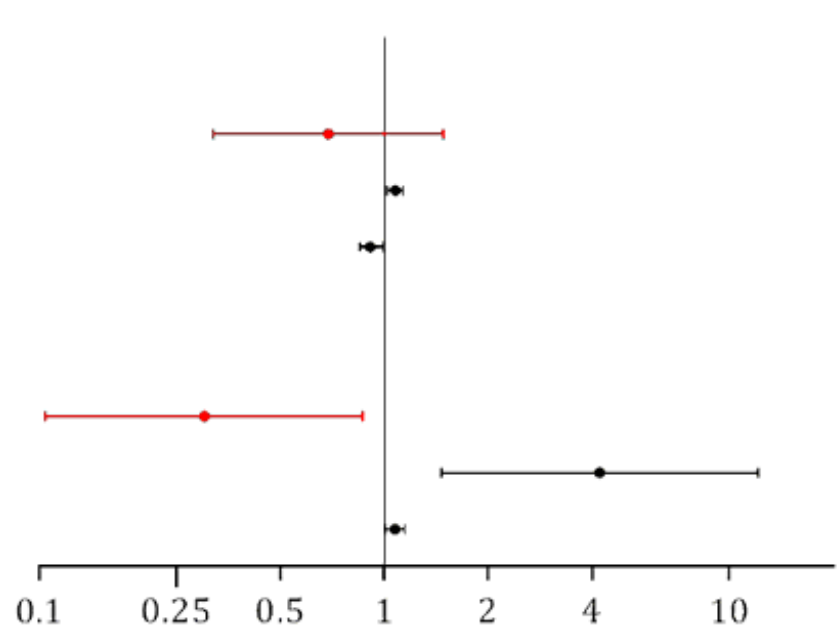

OR $(95 \% \mathrm{CI})$

$0.688(0.320-1.479)$

0.338

$1.078(1.023-1.132)$

0.003

$0.952(0.911-0.994)$

0.026

$0.301(0.104-0.867) \quad 0.026$

$4.207(1.461-12.111) \quad 0.008$

$1.073(1.066-1.146) \quad 0.034$

Figure 2

Independent influencing factor》IIF囚on PPD at $24 \mathrm{~h}$ post-delivery before and after propensity matching (PSM).

Factors

Original data $(n=263)$

Epidural analgesia

Anxiety and depression during pregnancy

Unplanned pregnancy

Matched by PSM $(\mathrm{n}=138)$

Epidural analgesia

Anxiety and depression during pregnancy

Unplanned pregnancy

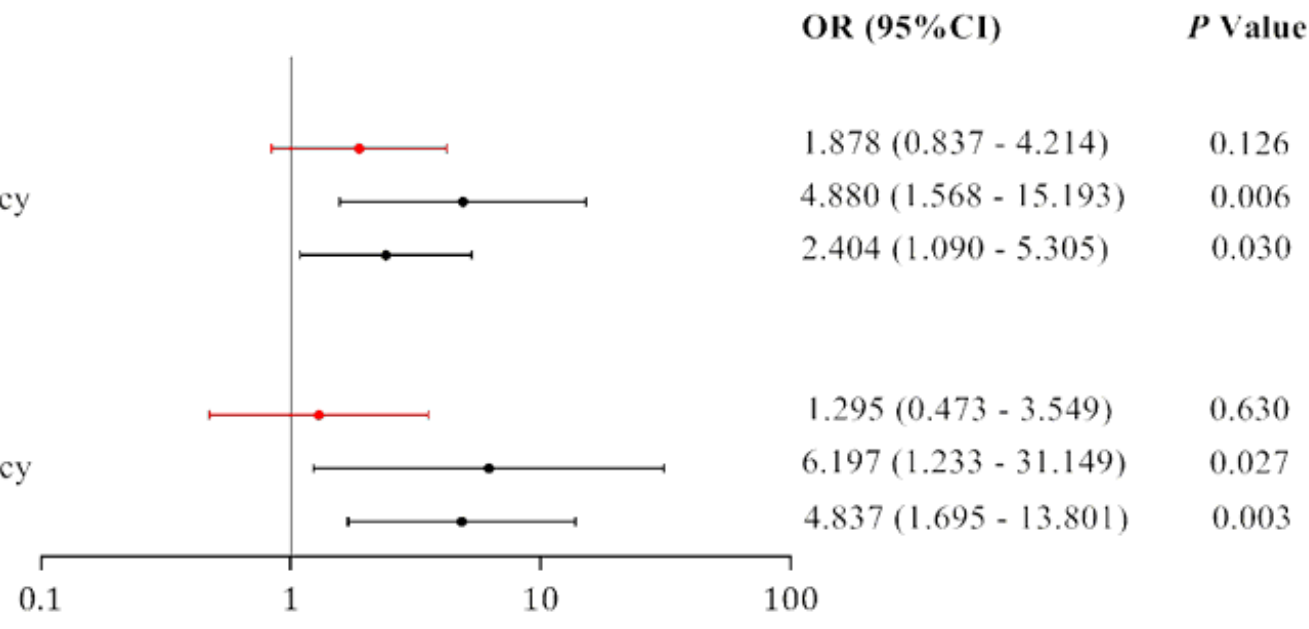

\section{Figure 3}

IIF on PPD at 42 days before and after PSM 


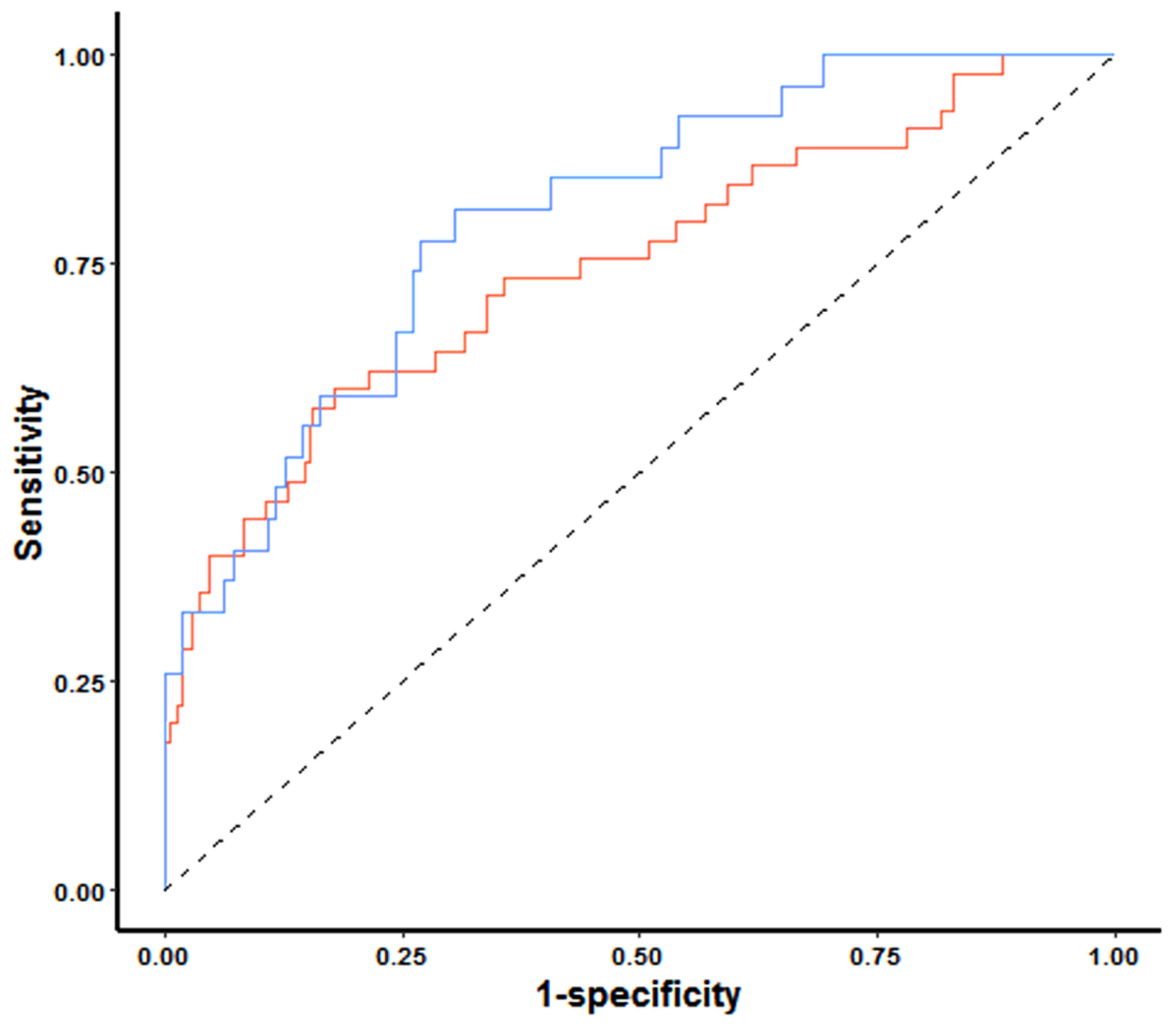

- Original data, AUC $=0.748(95 \% \mathrm{Cl}: 0.660$ - 0.835)

- Matched by PSM, AUC = 0.806 (95\% Cl: $0.718-0.895)$

Figure 4

ROC curves on PPD at $24 \mathrm{~h}$ post-delivery before and after PSM 


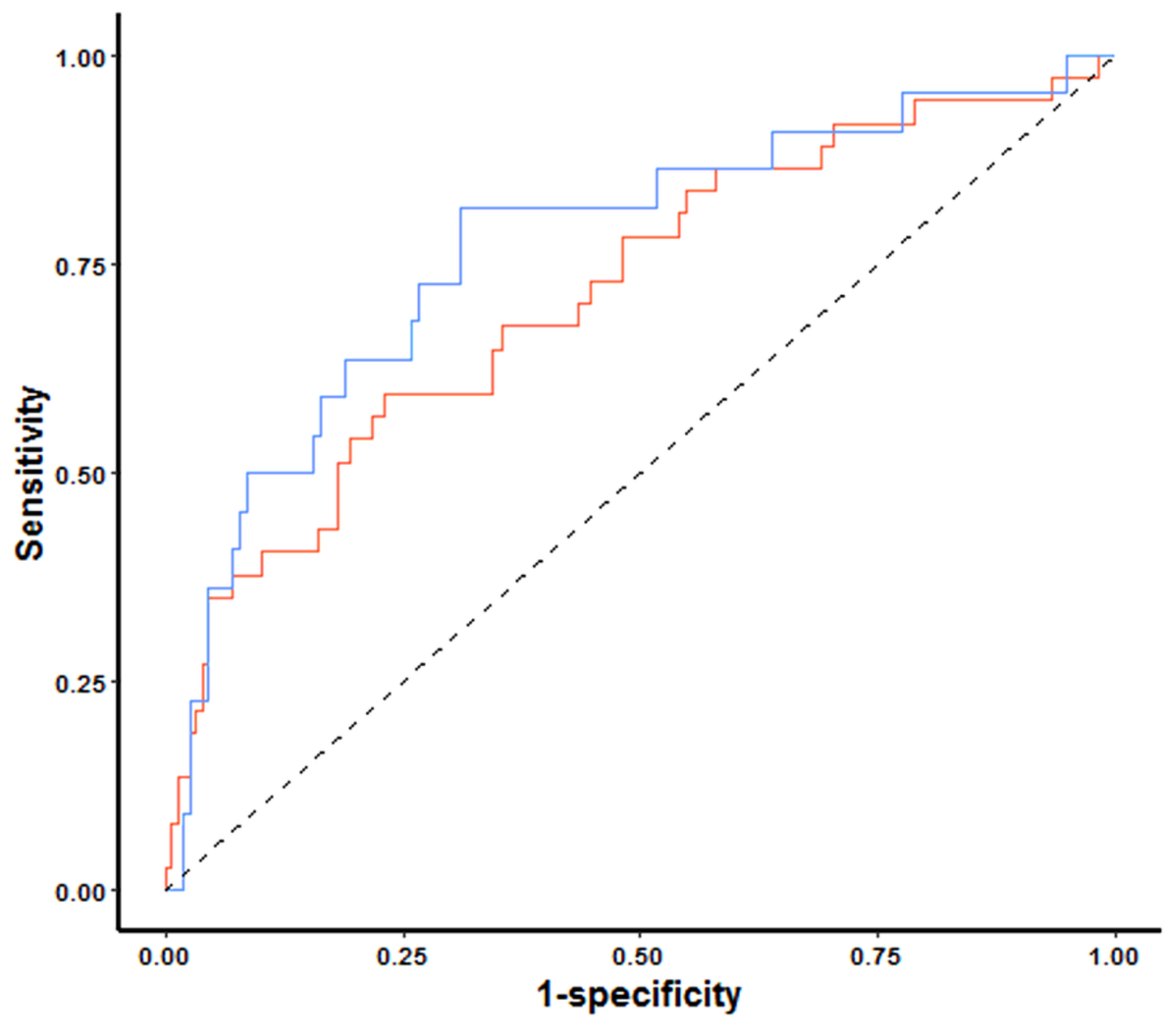

- Original data, AUC $=0.716(95 \% \mathrm{Cl}: 0.620$ - 0.812)

- Matched by PSM, AUC = $0.772(95 \% \mathrm{Cl}: 0.655-0.890)$

Figure 5

ROC curves on PPD at 42 days before and after PSM 
Factors

Original data $(n=263)$

Epidural analgesia

Maternal blood glucose control mode

Matched by PSM $(n=138)$

Epidural analgesia

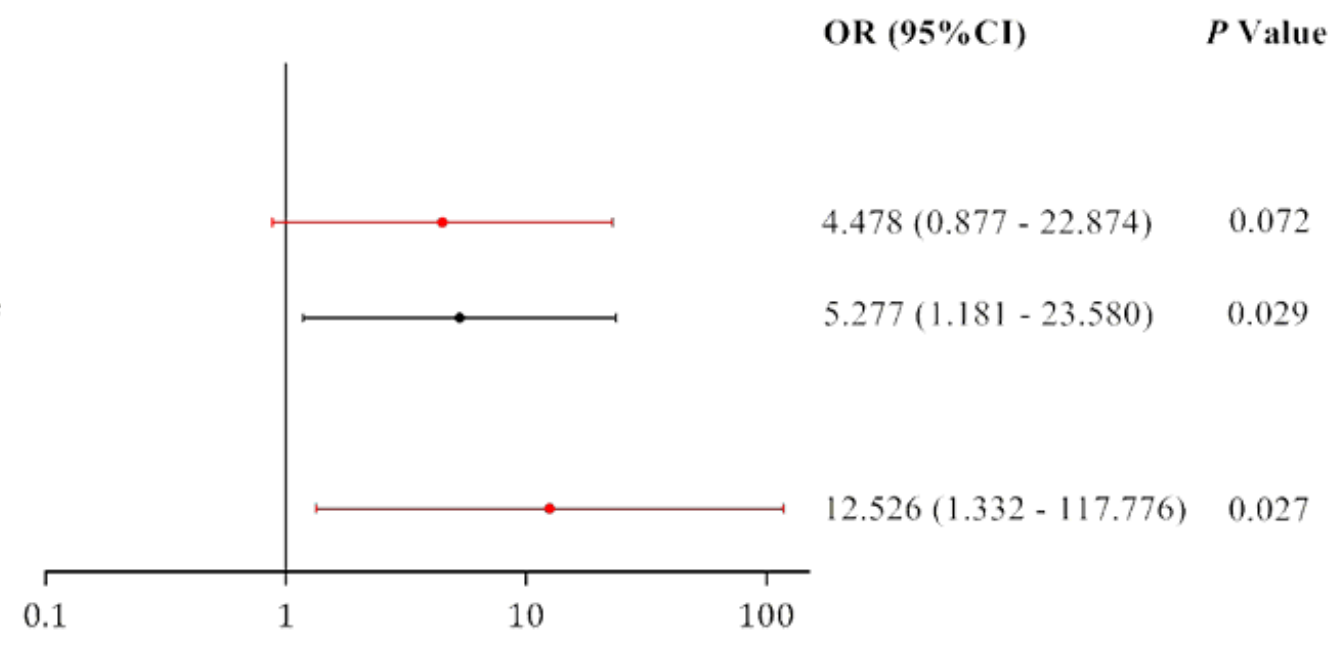

\section{Figure 6}

IIF on the rate of neonate transfer to the NICU for hypoglycemia treatment before and after PSM 


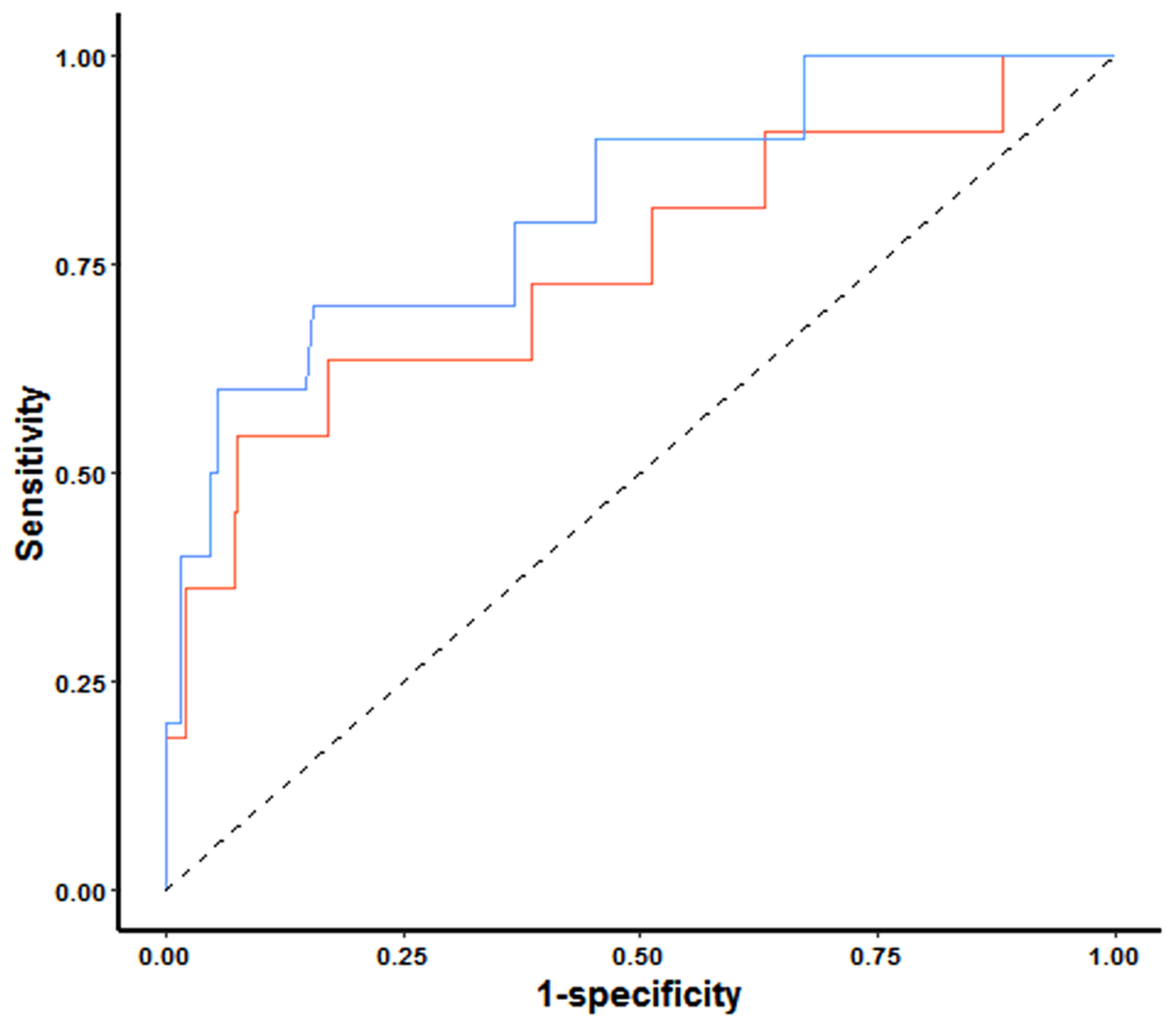

- Original data, AUC $=0.749(95 \% \mathrm{Cl}: 0.567-0.930)$

- Matched by PSM, AUC $=0.822(95 \% \mathrm{Cl}: 0.672$ - 0.972)

Figure 7

ROC curves on the rate of neonate transfer to the NICU for hypoglycemia treatment before and after PSM 